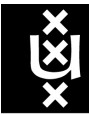

UNIVERSITEIT

VAN AMSTERDAM

\title{
Bayesian Inference for the A/B Test: Example Applications with JASP
}

\author{
Master Thesis
}

\section{Tabea Hoffmann}

(12341320)

\section{Eric-Jan Wagenmakers}




\section{Bayesian Inference for the A/B Test: Example Applications with JASP}

Most popular in clinical studies, but also the latest trend in business, A/B testing is a procedure to compare the success frequencies of two alternatives, such as two treatment arms or two website versions. With the present paper, we advocate for using a Bayesian approach to A/B testing. We demonstrate how Bayesian A/B tests allow practitioners to address various questions concerning a difference between two proportions. With the proposed analyses, practitioners can monitor and quantify their uncertainty as data accumulate.

\section{When to Use This Procedure}

Experimentation is omnipresent in medical studies. It is used, for example, to compare the treatment success of a new pharmacological drug to a placebo. In recent years, there has been a growing interest in a similar kind of experimentation in the web development context as it allows establishing an evidence-based approach to business. In general, the number of visitors that take action on a website is a key indicator of success. The more traffic a website has, i.e., the more people visit and navigate through a website, the more chances there are for the acquisition of new customers and the promotion of customer loyalty. However, the traffic on a website is only profitable for a company if it translates into meaningful customer actions such as signing up for a newsletter or buying something. In technical jargon, the number of website visitors that take a desired action is called conversion. The goal of online marketers is to have the highest possible conversion rate which denotes the conversion count divided by the total number of unique visitors. To lure customers into clicking, signing up or buying, online marketers have to equip a website with a compelling design (Gudigantala, Bicen, et al., 2016; Saleh \& Shukairy, 2010). Experimentation allows them to test whether their assumptions on effective website design translate into the desired conversion rates.

In online marketing, an experiment may proceed as follows: Two versions of the 
same webpage are shown to different selections of website visitors and the number of visitors who take the desired action (e.g., the click rate) is monitored in a nominal dichotomous manner. The two website versions only differ in one aspect (e.g., the colour of a call-to-action button). Changing only one aspect ensures being able to identify what had an effect on website visitors' behaviour. Figure 1 illustrates the procedure. Eventually companies want to test which webpage version has the highest conversion rate. To make this judgment, the company needs to determine whether and to what extent the observed sample difference in proportions translates to the population; in other words, the company needs to conduct a statistical analysis.

\section{Figure 1}

A/B testing procedure for conversion rate optimization. Subdivisions of website visitors are redirected to either of two website versions. A variable of interest is measured and indicated as the conversion rate (number of conversions/total number of website visitors).

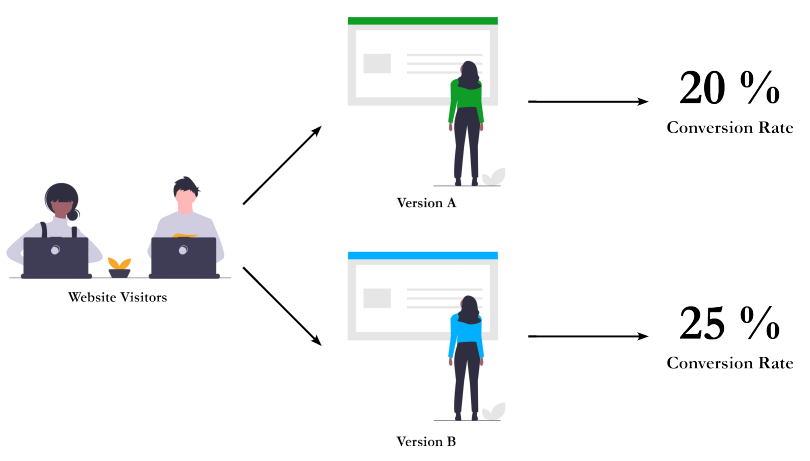

While data-driven decision-making sounds compelling to many businesses, it needs to be carried out correctly to yield the desired monetary pay-off. In the next section, we will introduce different statistical approaches that practitioners can adopt.

\section{Different Statistical Approaches to A/B testing}

The difference between the two sample proportions can be analyzed with an A/B test (Little, 1989). Ideally this test should meet three general desiderata (Gronau et al., 2019): evidence can be obtained in favor of the null hypothesis, evidence can be monitored 
as the data accumulate, and expert knowledge can be taken into account. In general, practitioners can chose between the frequentist and the Bayesian framework. With the desiderata in mind, we can evaluate the qualities of these two statistical approaches to the A/B test analysis. The content and meaning of the desiderata will become more explicit in the subsequent paragraphs.

\section{Frequentist Statistics}

As it is the case for many other analyses in various fields, online marketers predominantly utilize the p-value based null hypothesis significance testing (NHST). The NHST approach however does not satisfy the three desiderata. Firstly, the results do not distinguish between absence of evidence and evidence of absence. While evidence of absence denotes that an effect truly does not exist (e.g., two website versions do not differ in conversion rates), absence of evidence means that one cannot know whether there is an effect or not (i.e., the data are inconclusive) (Altman \& Bland, 1995). Secondly, the data cannot be tested sequentially. In practice, sellers need to know as quickly as possible which online campaign creates the highest conversion rate because many offers are bound to specific events such as Christmas shopping offers. To be time-efficient, many A/B test applicants repeatedly 'peek' at the results and call the testing quits as soon as the p-value is smaller than some predefined $\alpha$-level (Goodson, 2014). However, repeatedly monitoring the uncorrected p-value and stopping the analysis when significant results are achieved invalidates a NHST analysis (Wagenmakers, 2007): With an increasing number of tests, the probability that at least one of the tests is significant also increases, leading to an inflated type I error rate (Jennison, Turnbull, et al., 1990). A third disadvantage of p-value based A/B testing is that it does not allow incorporating expert knowledge. Among conversion rate optimization professionals it is widely known that online advertising campaigns often yield minuscule conversion increases (cf. Johnson et al., 2017; Patel, 2018). Enriching statistical models with such expert knowledge leads to more informed statistical 
analyses (Lindley, 1993).

\section{Bayesian Statistics}

The limitations of frequentist statistics can be overcome by adopting a Bayesian data analysis approach (e.g., Deng, 2015, Kamalbasha \& Eugster, 2020; Stucchio, 2015). In Bayesian statistics, probability expresses a degree of reasonable belief (Jeffreys, 1961) and in principle Bayesian statistics fulfill all three desiderata (e.g., Wagenmakers et al., 2018). In recent years, some practitioners have discovered the benefits that Bayesian statistics bring for A/B testing (e.g., Deng, 2015, Kamalbasha \& Eugster, 2020 Stucchio, 2015). In the next section we introduce two approaches to Bayesian A/B testing. The two approaches make different assumptions, ask different questions, and therefore provide different answers.

\section{The 'Common Approach'}

Let $n_{A}$ denote the total number of observations and $y_{A}$ denote the number of successes for version $\mathrm{A}$. Let $n_{B}$ denote the total number of observations and $y_{B}$ denote the number of successes for version B. The commonly used Bayesian A/B testing model is specified as follows:

$$
\begin{aligned}
& y_{A} \sim \operatorname{Binomial}\left(n_{A}, \theta_{A}\right) \\
& y_{B} \sim \operatorname{Binomial}\left(n_{B}, \theta_{B}\right)
\end{aligned}
$$

This model assumes that $y_{A}$ and $y_{B}$ follow independent binomial distributions with success probabilities $\theta_{A}$ and $\theta_{B}$. These success probabilities are assigned independent beta $(\alpha, \beta)$ distributions that encode the relative prior plausibility of the values for $\theta_{A}$ and $\theta_{B}$. In a beta distribution, the $\alpha$ values can be interpreted as counts of hypothetical "prior successes" and the $\beta$ values can be interpreted as counts of hypothetical "prior failures" (Lee \& Wagenmakers, 2013):

$$
\begin{aligned}
& \theta_{A} \sim \operatorname{Beta}\left(\alpha_{A}, \beta_{A}\right) \\
& \theta_{B} \sim \operatorname{Beta}\left(\alpha_{B}, \beta_{B}\right)
\end{aligned}
$$


With the data coming in from the A/B testing experiment, the two independent prior distributions are updated to two independent posterior distributions. This is achieved by Bayes' rule:

$$
\begin{aligned}
p\left(\theta_{A} \mid y_{A}, n_{A}\right) & =\frac{p\left(\theta_{A}\right) \times p\left(y_{A}, n_{A} \mid \theta_{A}\right)}{p\left(y_{A}, n_{A}\right)} \\
p\left(\theta_{B} \mid y_{B}, n_{B}\right) & =\frac{p\left(\theta_{B}\right) \times p\left(y_{B}, n_{B} \mid \theta_{B}\right)}{p\left(y_{B}, n_{B}\right)}
\end{aligned}
$$

where $p\left(\theta_{A}\right)$ and $p\left(\theta_{B}\right)$ are the prior probabilities and $p\left(y_{A}, n_{A} \mid \theta_{A}\right)$ and $p\left(y_{B}, n_{B} \mid \theta_{B}\right)$ are the likelihoods of the data given the respective parameter. Hence, the reallocation of probability from prior to posterior is brought about by the data: the probability of parameters that predict the data well increases while the probability of parameter values that predict the data poorly decreases (Kruschke, 2013, van Doorn et al., 2020; Wagenmakers et al., 2016). Note that whenever a beta prior is used and the observed data has a binomial distribution, the resulting posterior distribution is also a beta distribution. In fact, if the data contains $s$ successes and $f$ failures, the resulting posterior beta distribution equals beta $(\alpha+s, \beta+f)$ (Gelman et al., 2013; van Doorn et al., 2020). 1 Eventually, practitioners are most often interested in the difference $\delta=\theta_{A}-\theta_{B}$ between the success rates of the two website versions. That is because this difference indicates whether the implemented changes on the website had the desired effect. In the next section, we will introduce an open source software implementation of the 'common approach.'

\section{Software Implementation of the 'Common Approach': The bayesAB Package}

The 'common approach' is implemented in the bayes AB (Portman, 2017) package in $\mathrm{R}$ ( $\mathrm{R}$ Core Team, 2020). Consider the following fictitious example. A company wishes to improve the click rate on their landing page. Therefore, they test two versions of this page - the original page and a modified version. Before setting up the A/B test and collecting the data, the prior distributions have to be specified so that they represent the relative

\footnotetext{
${ }^{1}$ When the prior and the posterior belong to the same family of distributions they are said to be conjugate.
} 
plausibility of the parameter values. For the present example we specify two uninformed beta(1,1) priors - a popular choice (Bondarenko \& Kravchenko, 2019; Singh, 2019). Each version is visited by $N=100$ people. In version A - the original website - 35 people click, and in version $\mathrm{B}$ - the modified website -50 people click. After running the A/B test procedure, the priors are updated with the obtained data. The $\mathrm{R}$ file for the fabrication of the data for this fictitious example can be found in the OSF repository associated with this manuscript (https://osf.io/anvg2/).

With bayesAB the calculation of the posterior distributions is done by feeding both the priors and the data to the bayesTest function:

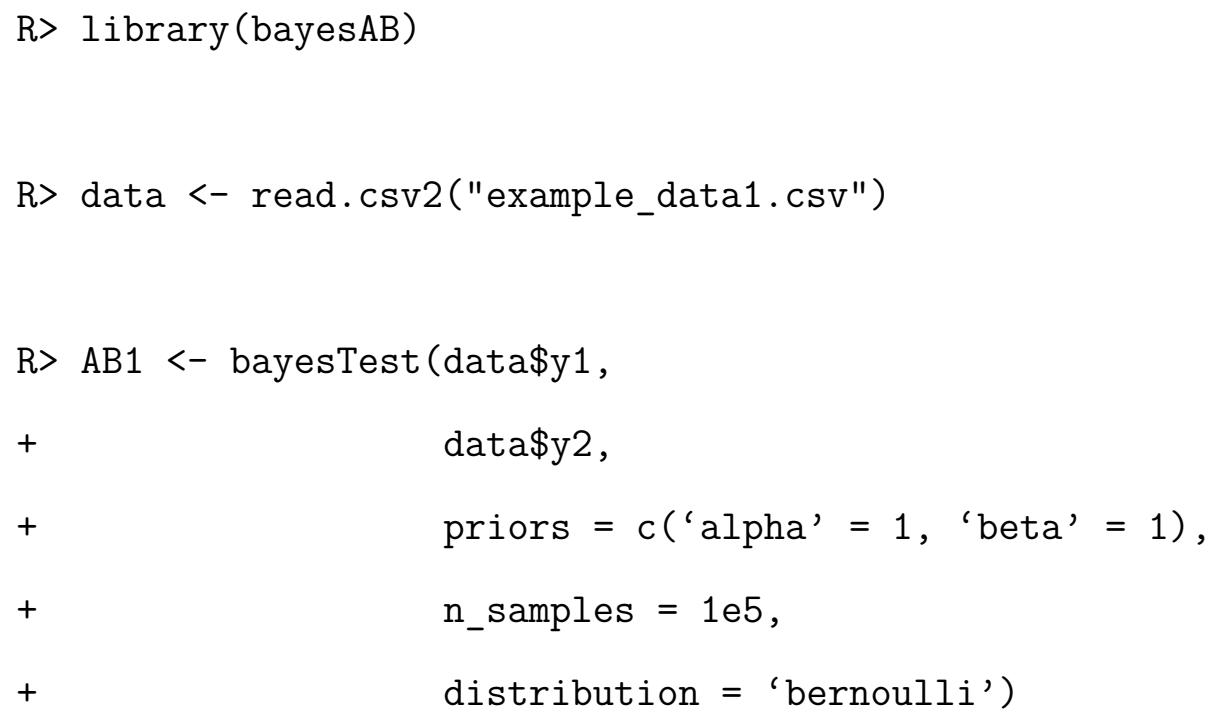

The results can be obtained and visualized:

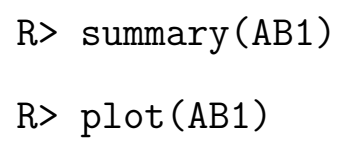

Figure 2 shows the two independent posterior distributions that plot (AB1) returns. To plot these posterior distributions, bayesTest makes use of the rbeta function that draws random numbers from a given beta distribution. To obtain each posterior distribution the package first exploits conjugacy: the number of successes $s$ are added to the $\alpha$ values of either version's prior distribution and the number of failures $f$ are added to 


\section{Figure 2}

Independent posterior beta distributions of the success probabilities for version $A$ and $B$. The plot is produced by the bayesAB package with the fictitious data. The analysis used two independent beta $(1,1)$ priors.

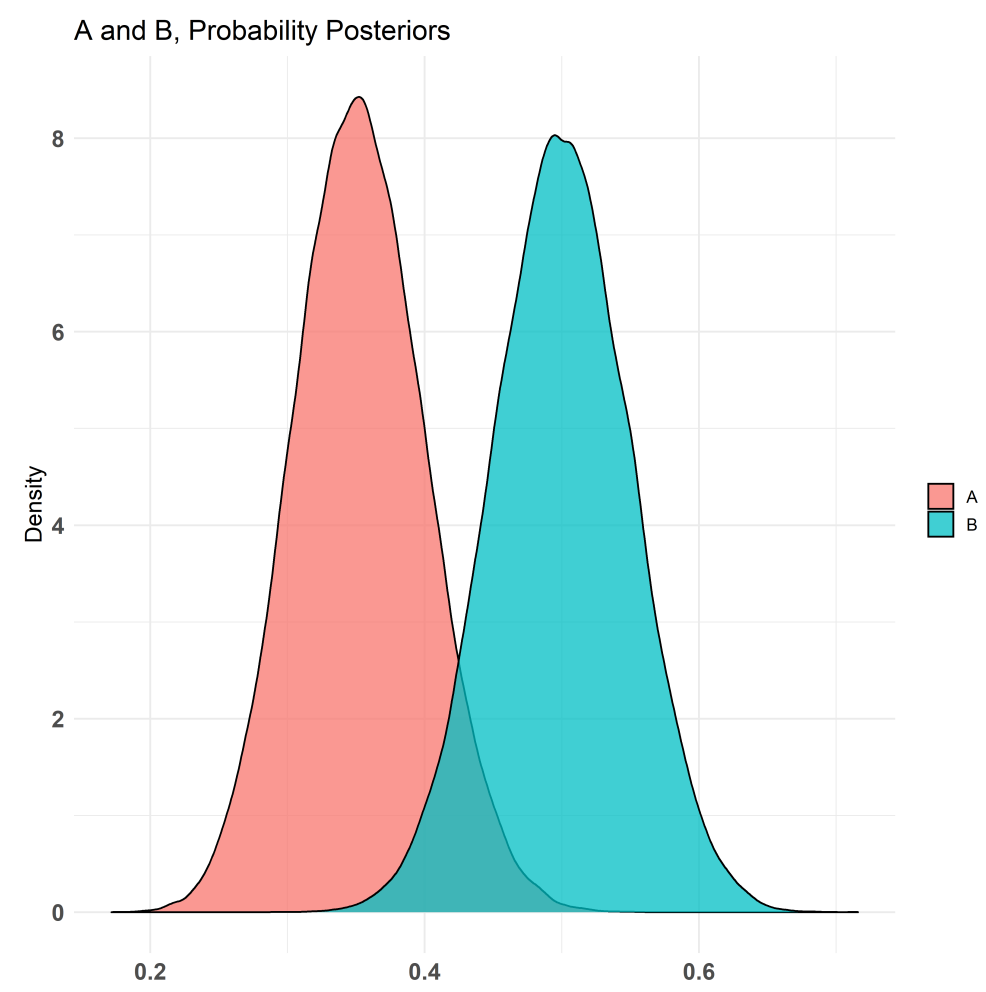

the respective $\beta$ values. Thus, the posterior distribution for $\theta_{A}$ is beta $\left(\alpha_{A}+s_{A}, \beta_{A}+f_{A}\right)$ and that for $\theta_{B}$ is beta $\left(\alpha_{B}+s_{B}, \beta_{B}+f_{B}\right)$. The rbeta function draws random samples from each posterior distribution and the density of these values is shown in Figure 2$]^{2}$ We can see that version B's posterior distribution for the success probability assigns more mass to higher values of $\theta$. This suggests that the success probability of the modified website version is higher and the alterations had a positive impact on the click rate.

The bayesAB package also returns a posterior distribution (see Figure 3) which

\footnotetext{
2 The posterior distributions are available analytically, so at this point the rbeta function is not needed; it will become relevant once we start to investigate the posterior distribution for the difference between $\theta_{A}$ and $\theta_{B}$.
} 
indicates the conversion rate uplift. The uplift is calculated as: $\frac{\left(\theta_{B}-\theta_{A}\right)}{\theta_{A}}$; that is, it is the difference between the success rates (i.e., $\theta_{B}-\theta_{A}$ ) expressed as a proportion of $\theta_{A}$. This posterior distribution is computed from the random samples obtained for the two beta posteriors shown in Figure 2.

The posterior distribution for the uplift peaks at around 0.4, indicating that the most likely conversion increase equals $40 \%$. Also, most posterior mass (i.e., $98.4 \%$ of the samples) is above zero, indicating that we can be $98.4 \%$ certain that version B is better than version A. Note that this statement assumes that it is equally likely that the alterations in versions B had a positive or negative effect on the conversion rate!

\section{Figure 3}

Histogram of the conversion rate uplift from version A to version B. The uplift is calculated by dividing the difference in conversion by the conversion in A. The plot is produced by the bayesAB package.

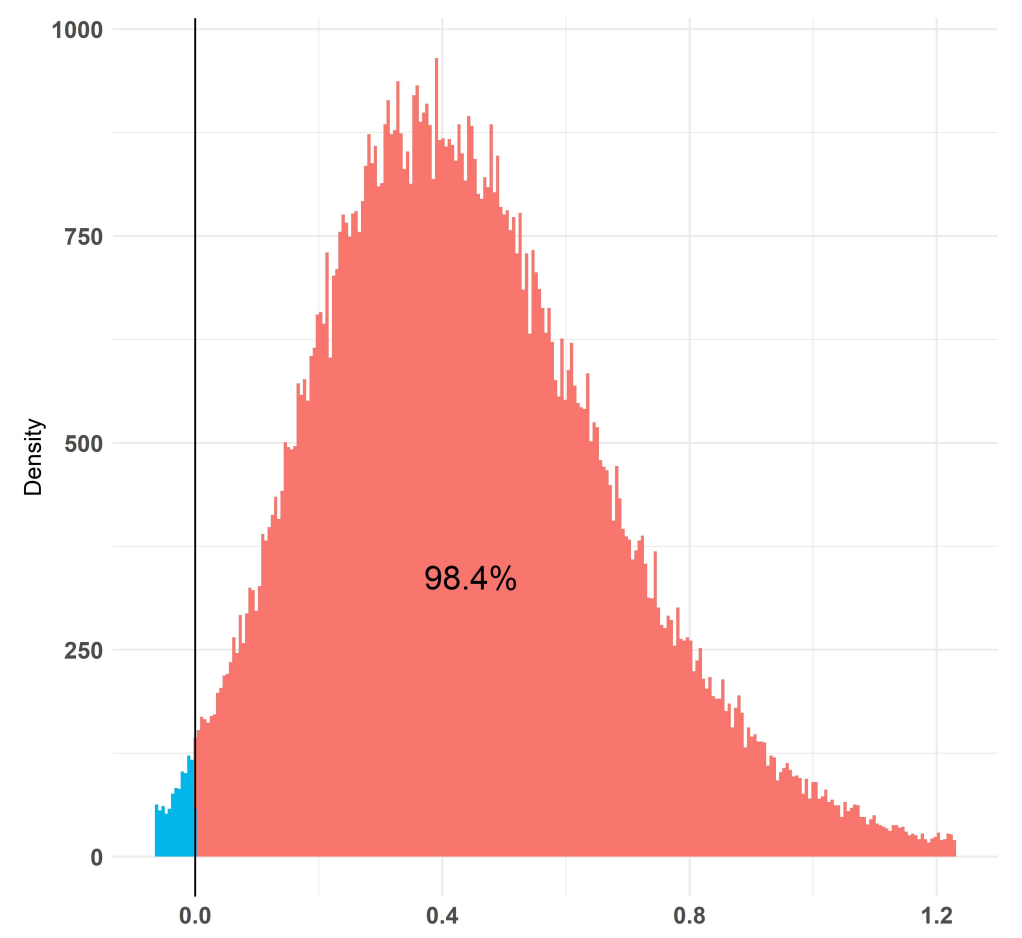

The posterior probability for $\theta_{B}>\theta_{A}$ can also be calculated analytically (Schmidt \& Mørup, 2019). The formula is not implemented in the bayesAB package; our $\mathrm{R}$ 
implementation can be found in the OSF repository. For the above example, $p\left(\theta_{B}>\theta_{A} \mid\right.$ data $)=0.984$. As far as the posterior distribution for the difference between the two independent beta distributions is concerned, it can be calculated analytically as well (Pham-Gia et al., 1993). Figure 4a shows the posterior density of the difference between the two independent beta posteriors from the above example. Unfortunately, the analytic calculation fails for values of $\alpha$ and $\beta$ above $\sim 50$, which occur with strong advance knowledge or high sample sizes. In this case, one can instead employ a normal approximation, the result of which is shown in the right panel of Figure 4$]^{3}$ Note however that this is only a crude approximation of $\delta$. For a detailed explanation and an alternative approximation see Appendix B.

\section{Figure 4}

Posterior density distributions of the difference $\theta_{B}-\theta_{A}$ for the fictitious example data.

(a) Analytically calculated beta difference distribution.

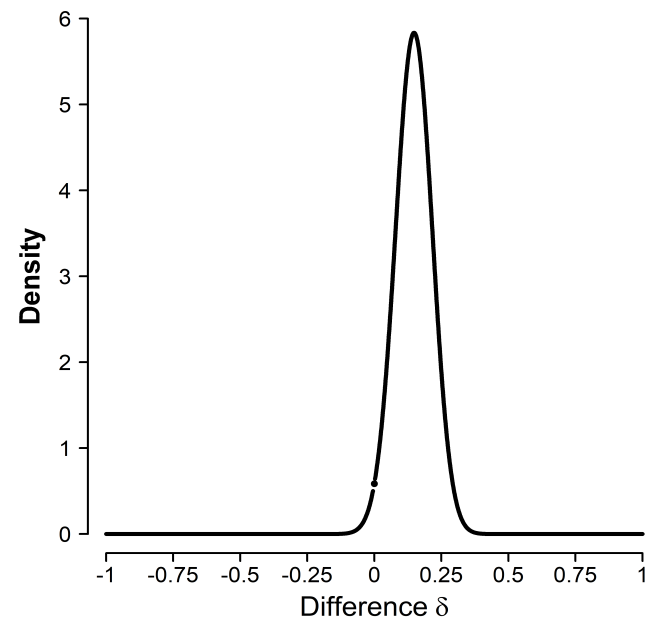

(b) Normal approximation of the difference between two independent beta distributions.

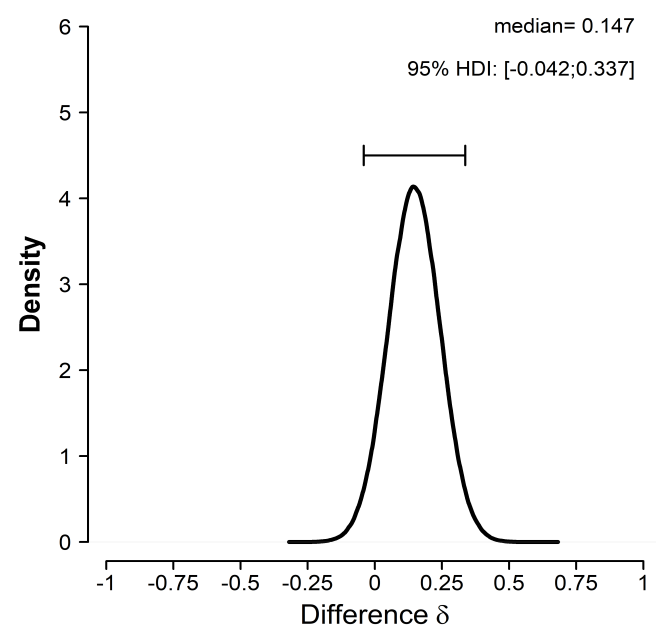

An advantage of the Bayesian approach is that the data can also be added to the analysis in a sequential manner. This means that the evidence can be assessed continually ${ }^{3}$ Appendix A contains the formulas from Schmidt and Mørup (2019) and Pham-Gia et al. (1993), as well as the formulas for the normal approximation. 
as the data arrives and the analyses can be stopped as soon as the evidence is judged to be compelling (Deng et al., 2016). Figure 5 plots the posterior mean of the difference between $\theta_{A}$ and $\theta_{B}$ as well as the $95 \%$ highest density interval (HDI) of the difference in a sequential manner. With increasing sample size, the HDI becomes more narrow. This indicates that the range of likely values for $\delta$ becomes smaller. After some initial fluctuation, the posterior mean difference between $\theta_{A}$ and $\theta_{B}$ settles between 0.1 and 0.2 . To create this plot, we used the abtest package in $R$ which will be introduced in a later section.

Appendix B contains the $\mathrm{R}$ code for the sequential computation.

\section{Figure 5}

Sequential analysis of the difference between the success probabilities (i.e., $\theta_{A}-\theta_{B}$ ) of the two website versions. The orange line plots the posterior mean of the absolute risk. The grey area visualizes the width of the highest density interval as a function of sample size $n$.

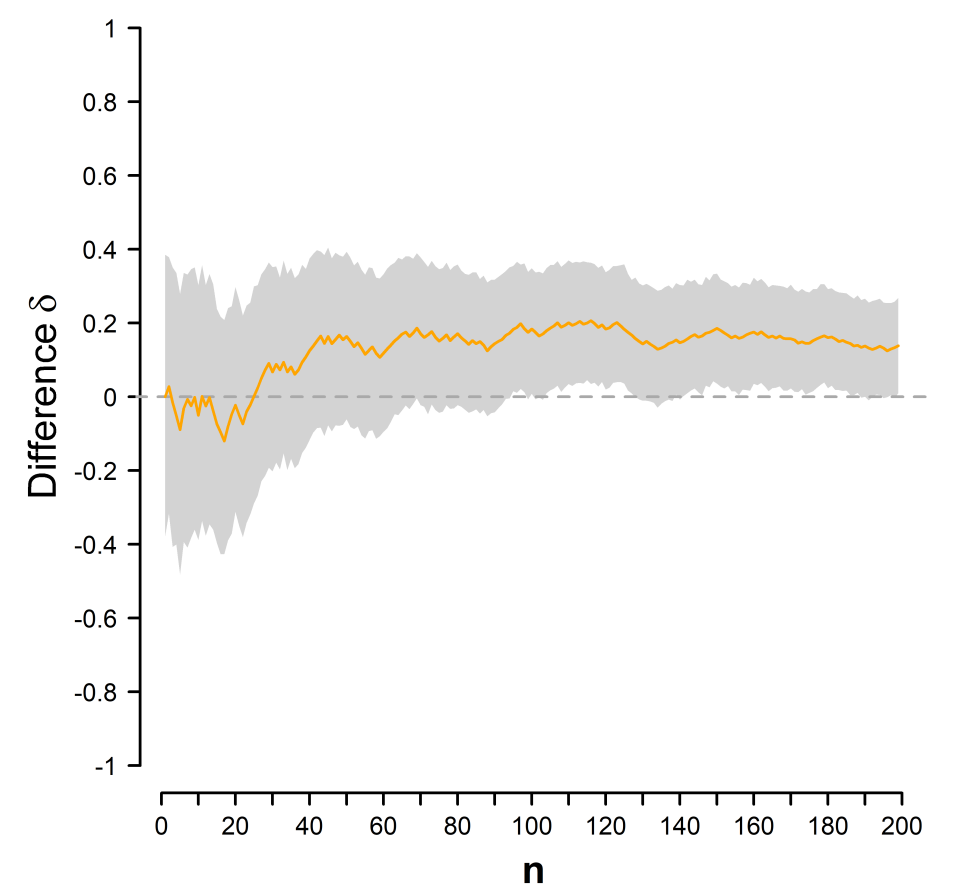

In sum, the common approach allows practitioners to judge the size and direction of an effect, that is, the difference between the two success probabilities. It is important, 
however, to recognise the assumptions that are made under this approach. In the next section, we will elaborate on these assumptions and their consequences.

\section{Assumptions of the 'Common Approach'}

The 'common approach' makes two important assumptions. The first assumption is that the two success probabilities are independent: learning about the success rate of one version does not affect our knowledge about the success rate of the other version (Howard, 1998). In practice, this assumption is rarely valid. If we know the conversion rate under version A relatively precisely, we anticipate that the conversation rate under version $\mathrm{B}$ is relatively close.

The second assumption of the common approach is that an effect is always present; that is, a new version may increase conversation rates or decrease conversion rates; it is never the case that the new version is completely ineffective. This assumption is a consequence of the fact that a continuous prior assigns zero probability to a specific point value such as zero (Jeffreys, 1939; Williams et al., 2017; Wrinch \& Jeffreys, 1921). Thus, using the common approach practitioners can only test whether website alterations yield a positive or negative effect. Obtaining evidence in favor of the null hypothesis - which was one of the desiderata listed by Gronau et al. (2019) - is not possible with this approach. Hence, 'the common approach' does not represent a testing effort, but an estimation effort (Jeffreys, 1939). To allow for both hypothesis testing and parameter estimation a Bayesian A/B testing model has to be able to assign prior mass to the possibility that the difference between the two versions is exactly zero. Next, we will introduce such an approach.

\section{The 'Odds Ratio Approach'}

An A/B test model that assigns prior mass to the null hypothesis of no effect was introduced by Kass and Vaidyanathan (1992) and implemented by Gronau et al. (2019). In contrast to the 'common approach', this model assigns a prior distribution to the log odds ratio, thereby accounting for the dependency between the success probabilities of the two 
website versions. The odds are the ratio of an event occurring to an event not occurring and the odds ratio quantifies the odds of an event occurring in one group compared to another (see Figure 6). The 'odds ratio approach' is specified as follows:

$$
\begin{gathered}
y_{A} \sim \operatorname{Binomial}\left(n_{A}, \theta_{A}\right) \\
y_{B} \sim \operatorname{Binomial}\left(n_{B}, \theta_{B}\right) \\
\log \left(\frac{\theta_{A}}{1-\theta_{A}}\right)=\gamma-\psi / 2 \\
\log \left(\frac{\theta_{B}}{1-\theta_{B}}\right)=\gamma+\psi / 2
\end{gathered}
$$

As before, this model assumes that $y_{A}$ and $y_{B}$ follow binomial distributions with success probabilities $\theta_{A}$ and $\theta_{B}$. However, the success probabilities are a function of two parameters, $\gamma$ and $\psi$. Parameter $\gamma$ indicates the grand mean of the log odds, while $\psi$ denotes the distance between the two conditions (i.e., the log odds ratio). The hypothesis that there is no difference between the two versions can be formulated as a null hypothesis: $\mathcal{H}_{0}: \psi=0$. Under the alternative hypothesis $\mathcal{H}_{1}, \psi$ is assumed to be nonzero. By default, both parameters are assigned normal priors:

$$
\begin{gathered}
\gamma \sim N\left(\mu_{\gamma}, \sigma_{\gamma}^{2}\right) \\
\psi \sim N\left(\mu_{\psi}, \sigma_{\psi}^{2}\right)
\end{gathered}
$$

While the choice of a prior for $\gamma$ is relatively inconsequential for the comparison between $\mathcal{H}_{0}$ and $\mathcal{H}_{1}$, the choice of a prior for $\psi$ is far-reaching: it determines the predictions of $\mathcal{H}_{1}$ concerning the difference between versions $\mathrm{A}$ and $\mathrm{B}$. For this reason, $\psi$ is the test-relevant parameter.

We consider four hypotheses that may be of interest to online marketers: $\mathcal{H}_{0}: \theta_{A}=\theta_{B} ;$ The success probabilities $\theta_{A}$ and $\theta_{B}$ are identical. $\mathcal{H}_{1}: \theta_{A} \neq \theta_{B} ;$ The success probabilities $\theta_{A}$ and $\theta_{B}$ are not identical. $\mathcal{H}_{+}: \theta_{B}>\theta_{A}$; The success probability $\theta_{B}$ is larger than the success probability $\theta_{A}$. 


\section{Figure 6}

Illustration of the odds and the odds ratio.

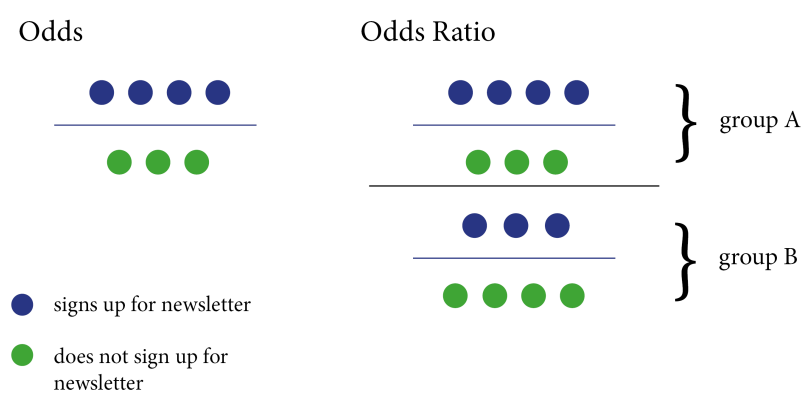

$\mathcal{H}_{-}: \theta_{A}>\theta_{B} ;$ The success probability $\theta_{A}$ is larger than the success probability $\theta_{B}$.

By comparing these hypotheses online marketers may answer the following questions: (1) Is there a difference between website versions $\mathrm{A}$ and $\mathrm{B}$, or are both versions equally effective? This requires a comparison between $\mathcal{H}_{1}$ and $\mathcal{H}_{0}$; (2) Does version B yield more successes than version A, or are both versions equally effective? This requires a comparison between $\mathcal{H}_{+}$and $\mathcal{H}_{0}$; (3) Does version A yield more successes than version B, or are both versions equally effective? This requires a comparison between $\mathcal{H}_{-}$and $\mathcal{H}_{0}$; (4) Does version B yield more successes than version A, or does version A yield more successes than version B? This is the question addressed by the 'common approach' discussed earlier, and it requires a comparison between $\mathcal{H}_{+}$and $\mathcal{H}_{-}$.

To compare the evidence that the observed data provide for and against the hypotheses we quantify and compare the models' predictive performance $4^{4}$ For two models, say $\mathcal{H}_{0}$ and $\mathcal{H}_{+}$, the comparison of their likelihoods for the observed data is known as the Bayes factor (Jeffreys, 1939, Kass \& Raftery, 1995; Wagenmakers et al., 2018):

$$
\mathrm{BF}_{+0}=\frac{p\left(\operatorname{data} \mid \mathcal{H}_{+}\right)}{p\left(\operatorname{data} \mid \mathcal{H}_{0}\right)}
$$

where $\mathrm{BF}_{+0}$ indicates the extent to which $\mathcal{H}_{+}$outpredicts $\mathcal{H}_{0}$.

\footnotetext{
${ }^{4}$ We use the terms 'model' and 'hypothesis' interchangeably.
} 
The evidence from the data is expressed in the Bayes factor, but to compare two hypotheses in their entirety, the a priori plausibility of the hypotheses needs to be considered as well. Bayes' rule describes how we can use the Bayes factor to update the relative plausibility of the two competing models after having seen the data (Kass \& Raftery, 1995):

$$
\underbrace{\frac{p\left(\mathcal{H}_{+} \mid \text {data }\right)}{p\left(\mathcal{H}_{0} \mid \text { data }\right)}}_{\text {posterior odds }}=\underbrace{\frac{p\left(\mathcal{H}_{+}\right)}{p\left(\mathcal{H}_{0}\right)}}_{\text {prior odds }} \times \underbrace{\frac{p\left(\text { data } \mid \mathcal{H}_{+}\right)}{p\left(\text { data } \mid \mathcal{H}_{0}\right)}}_{\mathrm{BF}+0} .
$$

The prior odds quantify the plausibility of the hypotheses before seeing the data, while the posterior odds quantify the plausibility of the two hypotheses after taking the data into account (Wagenmakers et al., 2018). The Bayes factor is the evidence - the change from prior to posterior plausibility brought about by the data.

\section{Software Implementation of the 'Odds Ratio Approach': The abtest Package}

To demonstrate the analyses with the 'odds ratio approach' we can use the abtest package (Gronau, 2019) in R ( $\mathrm{R}$ Core Team, 2020). It is important that a hypothesis is specified before setting up the A/B test (McFarland, 2012). For the previous example, it can be assumed that the alterations implemented on website version B were designed with the express purpose to lead to more clicks. Hence, from a Bayesian perspective, we may want to compare the directional hypothesis $\mathcal{H}_{+}$that website visitors in version $\mathrm{B}$ generate more clicks (not fewer clicks) against the null hypothesis $\mathcal{H}_{0}$ that there is no difference in the click rate between version A and B. The prior distribution for the log odds ratio under $\mathcal{H}_{+}$has to be specified before the data collection, too, as it quantifies how large the difference between the two success probabilities is expected to be. For the present example, we assume that there is little prior knowledge, which is why we use an uninformed parameter prior. For the log odds ratio the normal distribution with $\mu=0$ and $\sigma^{2}=1$ can be employed. Within the abtest package, the prior is specified using the elicit_prior function: 


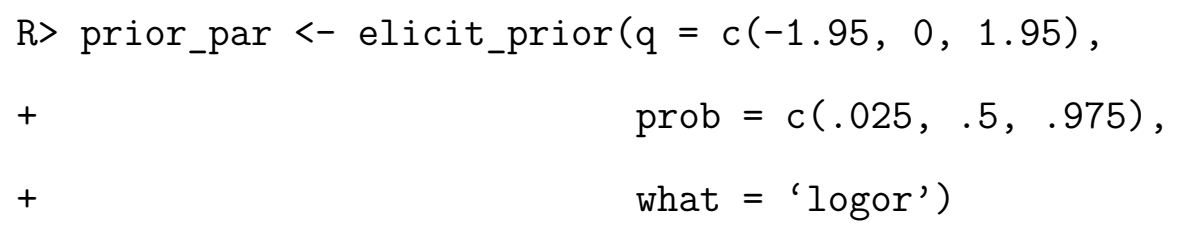

The observed success proportions of 35/100 for version A and 50/100 for version B suggest an enhanced click rate in version B as compared to version A. Whether this difference is statistically convincing needs to be assessed with a hypothesis test, for which we use the ab_test function. This function requires the data, parameter priors, and prior model probabilities. For the present example, we set the prior probabilities of $\mathcal{H}_{+}$and $\mathcal{H}_{0}$ to 0.5 :

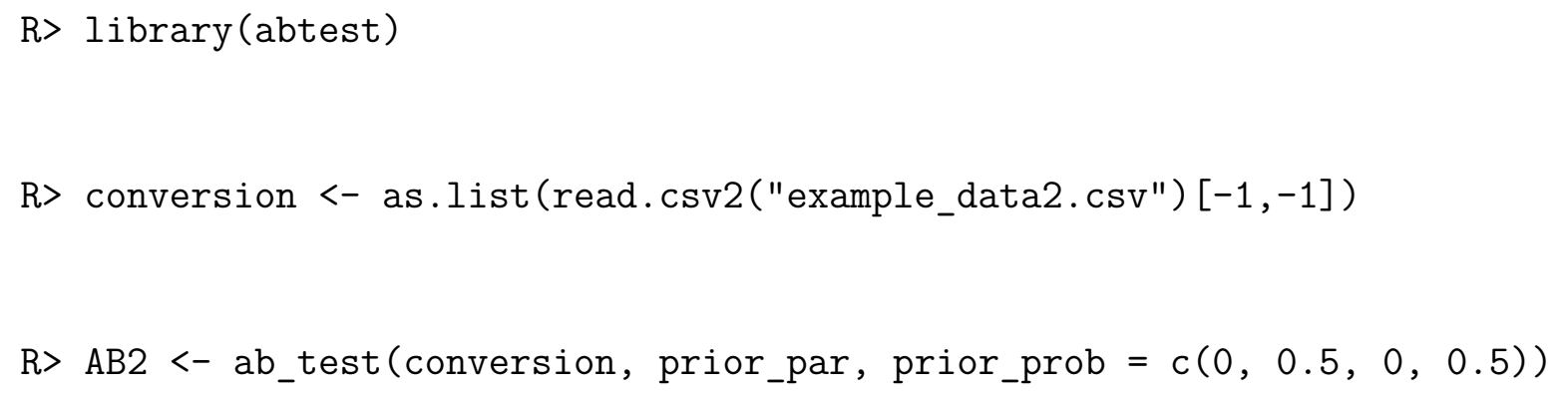

The function then returns the Bayes factors and the prior and posterior probabilities of the hypotheses. The Bayes factor $\mathrm{BF}_{+0}$ equals 4.7 , meaning that the data are approximately 5 times more likely to occur under the alternative hypothesis $\mathcal{H}_{+}$than under the null hypothesis $\mathcal{H}_{0}$. A Bayes factor of $\sim 5$ corresponds to moderate evidence (e.g., Jeffreys, 1939, Lee \& Wagenmakers, 2013).

The robustness of this conclusion can be explored by changing the prior distribution on $\psi$ (i.e., by varying the mean and standard deviation of the normal prior distribution) and observing the effect on the Bayes factor. Figure 7 visualizes the robustness of the Bayes factor for changes across a range of values for $\mu_{\psi}$ and $\sigma_{\psi}$. The Bayes factor is highest for low $\sigma_{\psi}$ values and $\mu_{\psi} \sim 0.6$. From looking at the heatmap we can deduce that our conclusion regarding the evidence for $\mathcal{H}_{+}$over $\mathcal{H}_{0}$ is relatively consistent. The plot can be produced with: 
$R>$ plot_robustness $(A B 2$, mu_range $=c(0,2)$, sigma_range $=c(0.1,1))$

\section{Figure 7}

Bayes factor robustness plot. The highest Bayes factor is reached for a prior on psi with $\mu_{\psi} \sim 0.6$ for the small example data set. Overall, the conclusion that there is moderate evidence for $\mathcal{H}_{+}$over $\mathcal{H}_{0}$ seems robust across a large range of values for $\mu_{\psi}$ and $\sigma_{\psi}$.

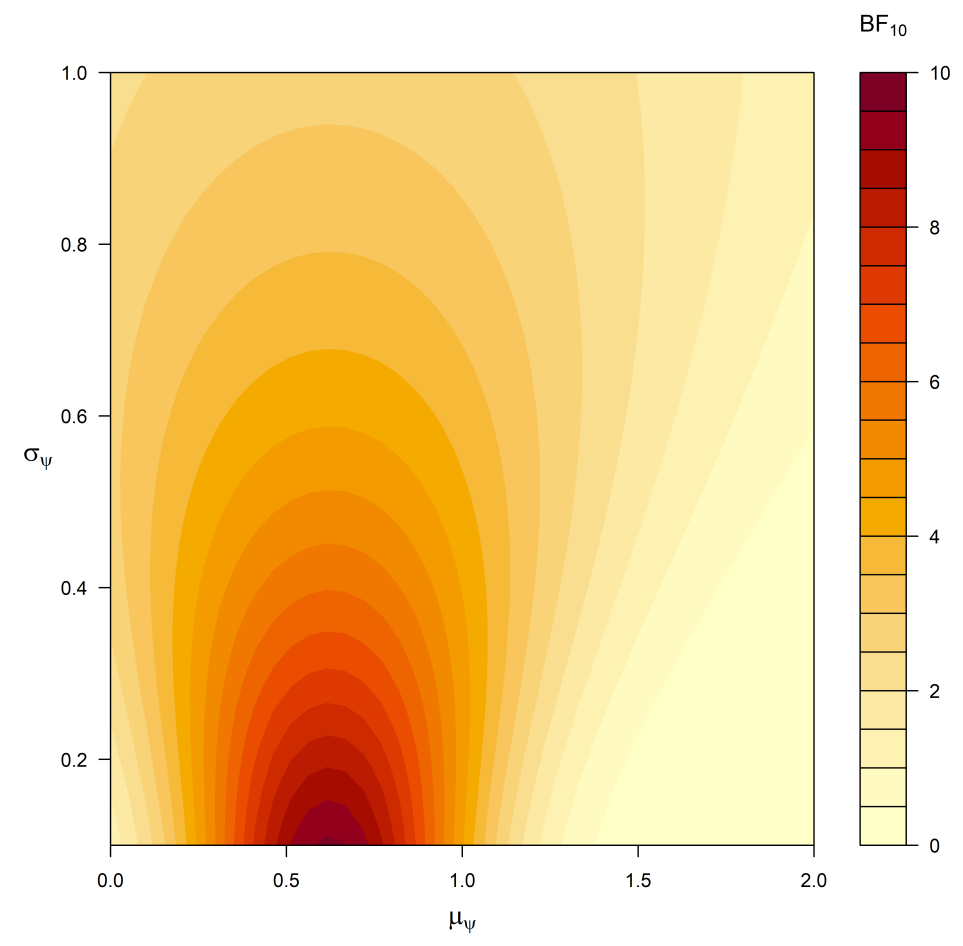

Figure 8 visualizes the posterior probabilities of the hypotheses as a probability wheel. The posterior probability of $\mathcal{H}_{+}$indicates that the data have increased the plausibility of the hypothesis from 0.5 to 0.823 while the posterior plausibility of $\mathcal{H}_{0}$ has correspondingly decreased from 0.5 to 0.177 .

The sequential analysis tracks the evidence in chronological order. Figure 9 plots the posterior probability of either hypothesis as a function of the number of observations. It shows that after some initial fluctuations, and a tie after about 40 observations, the last 160 observations cause the probability for the alternative hypothesis to increase substantially.

The sequential analysis as well as the probability wheel can be plotted as follows: 


\section{Figure 8}

Posterior probabilities of the hypotheses. The green area visualizes the posterior probability of the alternative hypothesis. The grey area visualizes the posterior probability of the null hypothesis. Both hypotheses were assigned a probability of 0.5 before testing.

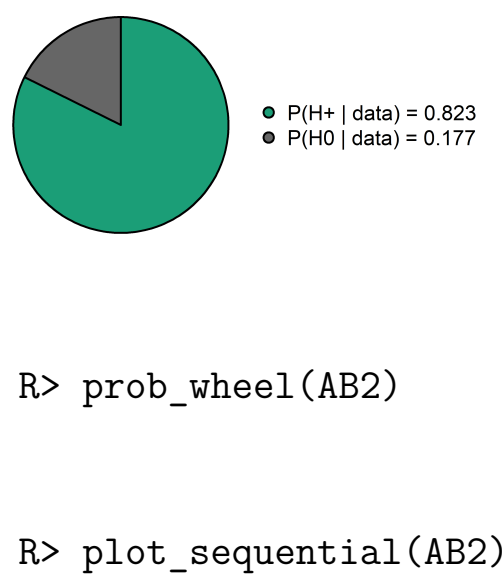

Having collected some evidence for a difference in effectiveness between the two versions, one might wish to then quantify this difference. To do so, estimation is required. We can again use Bayes' rule as described for the 'common approach' above.

Figure 10 shows the prior and posterior distribution for the log odds ratio under the two-sided model $\mathcal{H}_{1}$, as produced via the plot_posterior function:

$$
\mathrm{R}>\text { plot_posterior }(\mathrm{AB} 2 \text {, what }=\text { 'logor') }
$$

The dotted line in Figure 10 displays the prior distribution, the solid line displays the posterior distribution (with $95 \%$ central credible interval), and the posterior median and $95 \%$ central credible interval (CI) are displayed on top. For our fictional example, the plot indicates that, given that the log odds ratio is not exactly zero, it is $95 \%$ probable to lie between 0.027 and 1.124 , where the posterior median is 0.576 .

The posterior distributions for the two success probabilities can be inspected with:

$$
\mathrm{R}>\text { plot_posterior }(\mathrm{AB} 2 \text {, what }=\text { ' } \mathrm{p} 1 \mathrm{p} 2 \text { ') }
$$




\section{Figure 9}

The flow of posterior probability for $\mathcal{H}_{0}$ and $\mathcal{H}_{+}$as a function of the number of observations across both fictional website versions.

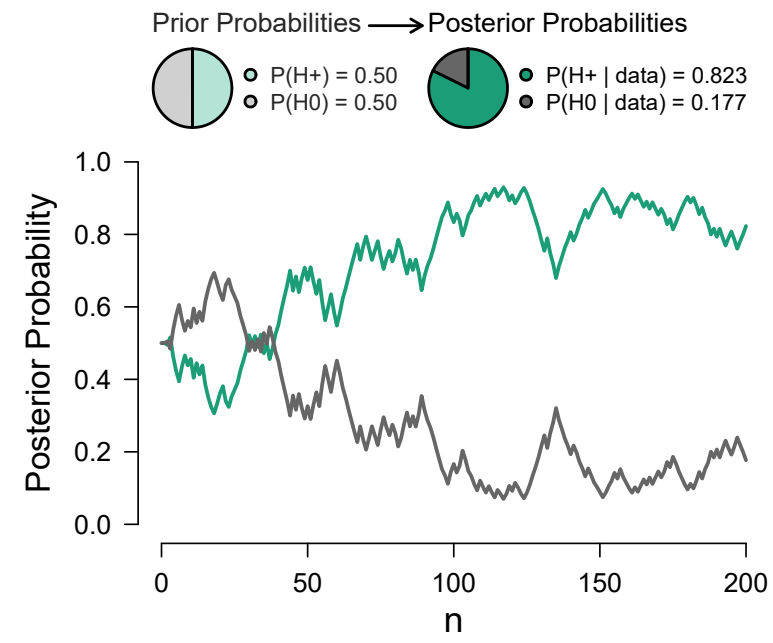

To showcase the different approaches to Bayesian A/B testing we carried out the analyses with two data sets. The first data set is a real data set that has been collected on an online learning platform. The second data set is fictitious. It serves the purpose to demonstrate the analyses with a data set that is very similar to data sets that result from conversion rate optimization efforts. We first report on the results that we acquired with the real data set. Afterwards, we will describe the analyses that we carried out with the second data set.

\section{Example 1: The Rekentuin Data Set}

\section{Preregistration}

The data has already been collected by Abe Hofman and colleagues on the Rekentuin website (data property of Oefenweb.nl) in 2019. To preregister our analyses we created data, carried out all analyses before receiving the real data, and stored the analysis scripts on our repository at the Open Science Framework (OSF). All preregistration 


\section{Figure 10}

Prior and posterior distribution of the log odds ratio under $\mathcal{H}_{1}$. The median and the $95 \%$ credible interval of the posterior density are shown in the top right corner.

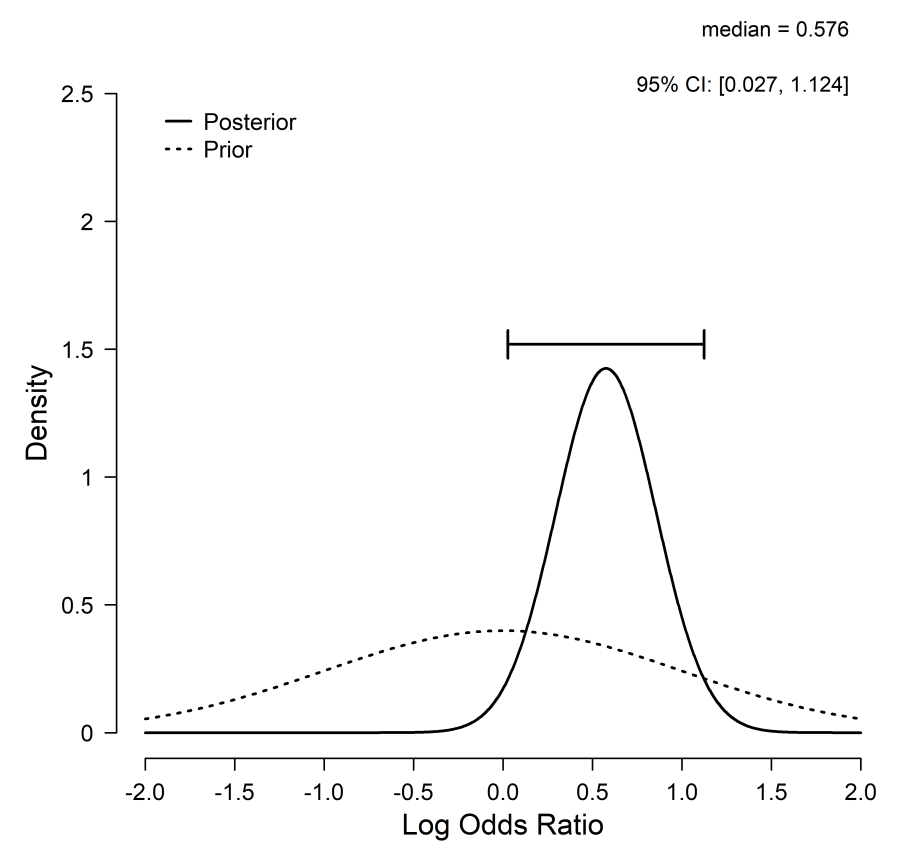

materials as well as the real data are available on the OSF repository at https://osf.io/anvg2/. Neither author of the present document has inspected the real data before the preregistration was finished. Conducting the analyses this way helped us avoid cognitive biases and other scientifically undesirable self-deception (Munafò et al., 2017). In the next section, we describe the Rekentuin data set.

\section{The Rekentuin Data Set}

Rekentuin is a tutoring website where children can playfully improve their mathematical skills with challenging online games. The children that are active on the Rekentuin website are Dutch elementary school children between the ages of 4 and 12 . During the testing interval from the $22^{\text {nd }}$ of January 2019 to the $5^{\text {th }}$ of February 2019 15,322 children were active on Rekentuin. 
Figure 11 shows a screenshot of the Rekentuin landing page. The basic principle is that by solving small math problems the children can earn coins (see the bottom right corner of Figure 12). With the coins, the children can buy virtual awards and add them to their trophy cabinet. The better a given student performs, the more trophies can be collected. The prospect of earning trophies motivates the children to perform better at the different math problems.

\section{Figure 11}

Example of the Rekentuin landing page. The figure shows that the child has earned three crowns for the category 'optellen' (Dutch for 'summation').

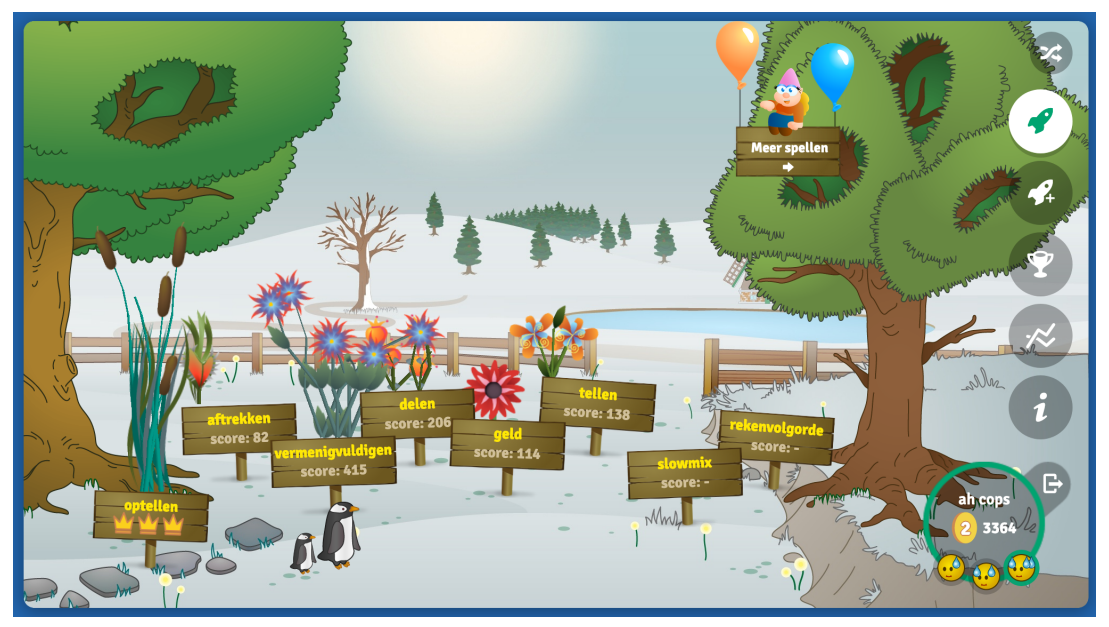

In 2019, the developers of Rekentuin were facing the challenge that many children would repeatedly solve math problems they were already performing well on. To incentivise the children to improve other skills (i.e., play other games), the developers implemented a crown for the games that the children had already mastered (Figure 11). More precisely, the crowns were created with the ulterior motive to prompt the children to collect crowns on as many games as possible. The players could gain up to a maximum of three crowns for each game. Thus, to obtain a crown for every game category the children had to engage more frequently with games they played less often. However, the crowns did not have the desired effect - instead of decreasing the playtime on crown games, the playtime on these crown games increased. 


\section{Figure 12}

Example of a game in the Rekentuin web environment. The coins are displayed on the bottom right corner.

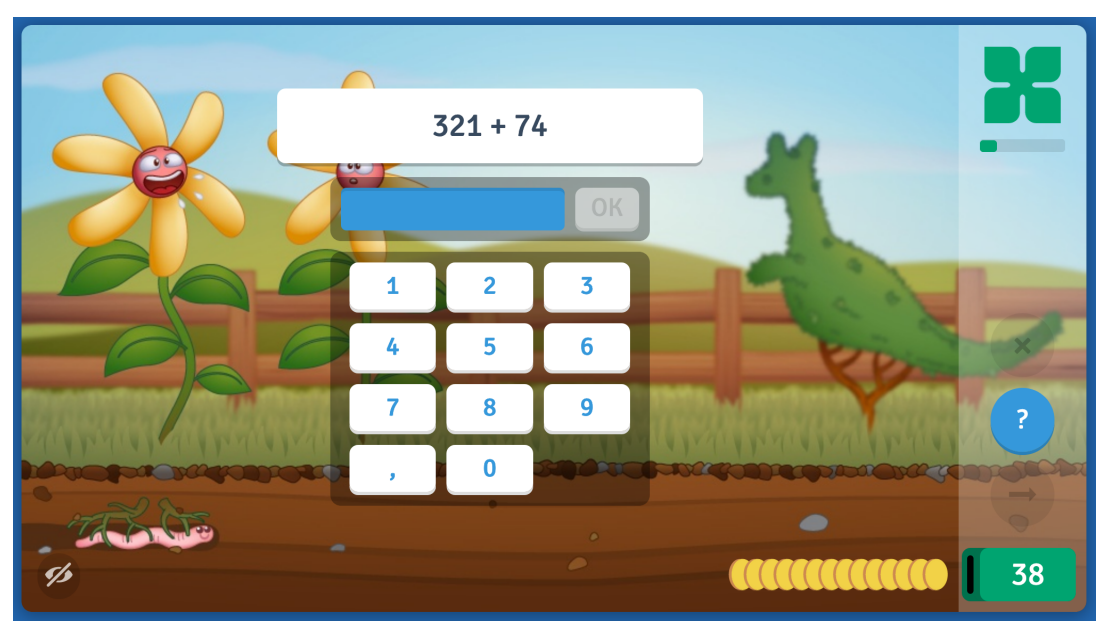

To induce the children to play other games, the developers constructed a less subtle manipulation: to take away the virtual reward (i.e., the coins) from the crown games. To test the effectiveness of this manipulation the Rekentuin developers designed an A/B test. One-half of the children continued playing on an unchanged website (version A), whereas the other half could no longer earn coins for crown games (version B). The children playing version $\mathrm{B}$ were not notified of the change but had to discover the changes for themselves.

\section{Results}

We applied the two Bayesian A/B testing approaches outlined above to the Rekentuin data set. In doing so we followed the general procedures discussed above. However, we used JASP for the 'odds ratio approach' analysis (JASP Team, 2020). JASP is an easy to use, free, open-source graphical program for statistical analysis. The program uses the abtest package in $\mathrm{R}$ as a back end. 5

As a dependent variable, we chose the last game the children played during the time

${ }^{5}$ For a detailed explanation on how to conduct the analyses in JASP read https://jasp-stats.org/2020/01/14/how-a-simple-bayesian-test-could-have-rescued-a-famous-clinical-trial/ 
of testing. We did so because we assumed that it will only become apparent after some time whether the children moved on to playing non-crown games. They might have only realized after some games that they do not receive any more coins for crown games.

Additionally, they might have hoped that the coins would reappear and therefore did not abandon their favourite game immediately. Thus, to obtain a binary variable, we noted for each player whether or not the last game played was a crown game.

We excluded players from the analyses according to two criteria. Firstly, players who did not play at least one crown game during the time of testing were excluded because they could not have experienced the experimental manipulation in version B. There were 8573 players in the data set who did not play at least one crown game during the testing interval. Secondly, we excluded 350 players who only played one crown game and it was their last game, because for these players we can not observe the potential influence of the manipulation on their playing behavior. In sum, we excluded 8923 players.

The question that needs to be answered is whether changing the incentive structure for crown games (i.e., removing the coins) had the desired effect. From a Bayesian perspective, we want to compare the directional hypothesis $\mathcal{H}_{+}$that children in version $\mathrm{B}$ played more non-crown games against the null hypothesis $\mathcal{H}_{0}$ that there is no difference in the amount of non-crown games between the two versions. For the 'common approach' we employed two uninformed beta distributions for the success probabilities of version A and B. For the 'odds ratio approach' we employed a truncated normal distribution with $\mu=0$ and $\sigma^{2}=1$ under the alternative hypothesis as there is a range of parameter values that seem plausible (see, for example, Cameron et al., 2001; Tang \& Hall, 1995).

\section{Descriptives}

Table 1 is a $2 \times 2$ table of the Rekentuin data where A refers to the control condition and $\mathrm{B}$ to the experimental condition. The table indicates the proportion of children that played a non-crown game as their last game. It seems as if the children in 


\section{Table 1}

Descriptives of the original Rekentuin data. Apparently children in version A played more non-crown games. The conditions (i.e. A and B) were switched for the statistical analyses as this data pattern seems unlikely.

\begin{tabular}{rrrr}
\hline & Counts & Total & Proportion \\
\hline A & 2596 & 3221 & 0.806 \\
B & 2272 & 3178 & 0.715 \\
\hline
\end{tabular}

version A played more non-crown games.

Having the strong manipulation in mind that was implemented on the Rekentuin website it seems unlikely to us that the children in the experimental condition B played less non-crown games. We think that the data was encoded incorrectly and that the real nature of the data is reversed. We assume that either the dependent variable or the conditions are interchanged in the data set. For our analyses we therefore switched the conditions: players that were originally indicated to be in the control condition A are now treated as if they are in the experimental condition $\mathrm{B}$ and vice versa.

\section{The 'Common Approach'}

Figure 13 displays the two independent posterior distributions. From Figure 13 we can see that version B's posterior distribution for the success probability assigns all mass to higher values of $\theta$ as compared to version A. This suggests that the success probability of the modified Rekentuin version is higher and that taking the coins away from the crown games had a positive impact on the amount of non-crown games that were being played.

Figure 14 indicates the conversion rate uplift. The distribution peaks at around 0.12 , indicating that the most likely conversion increase equals $12 \%$. Also, all posterior mass (i.e., $100 \%$ of the samples) is above zero. In other words, we can be $100 \%$ certain that version $B$ is better than version $A$. Note that this statement assumes that it is equally 


\section{Figure 13}

Independent posterior beta distributions of the success probabilities of playing a non-crown game. Version A corresponds to the unchanged Rekentuin website. Version B denotes the Rekentuin version where the players could not earn coins for crown games. The plot is produced by the bayesAB package. The analysis used two independent beta $(1,1)$ priors.

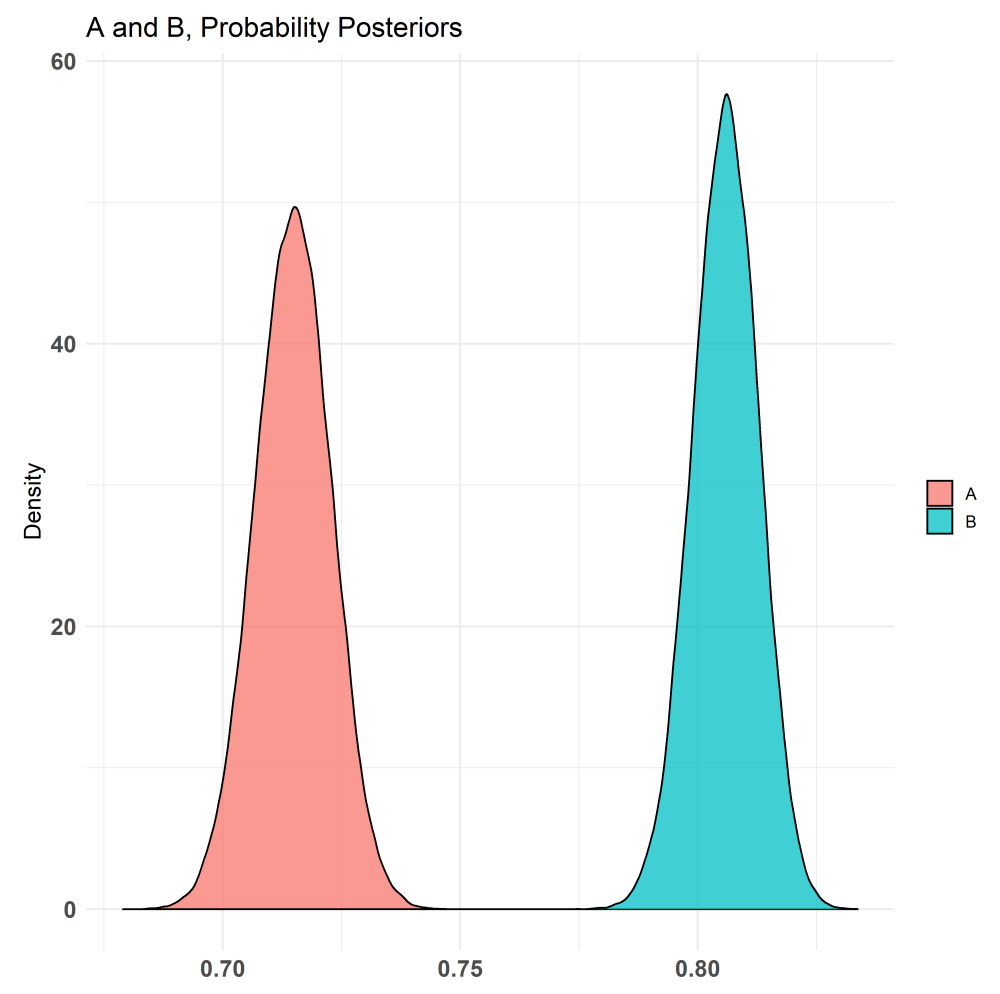

likely that the alterations in versions B had a positive or negative effect on the rate at which the children played non-crown games!

In addition to the bayesAB package output, we computed the posterior probability of the event $\theta_{B}^{\text {non-crown }}>\theta_{A}^{\text {non-crown }}$ using the formula reported by Schmidt and Mørup (2019). For the Rekentuin data $P\left(\theta_{B}>\theta_{A}\right)=1$. The analytic calculation of the posterior distribution for the difference $\delta=\theta_{B}^{\text {non-crown }}-\theta_{A}^{\text {non-crown }}$ between the two independent beta distributions fails for this data set because the data set is too large. We did not use the normal approximation of the difference as it is imprecise. Instead, we calculated the posterior samples of the absolute risk under $\mathcal{H}_{1}$. Figure 15 plots the entire probability 


\section{Figure 14}

Histogram of the conversion rate uplift from version $A$ to version $B$ of the Rekentuin website. The conversion rate indicates the proportion of players that played a non-crown game. The uplift is calculated by dividing the difference in conversion by the conversion in A. The plot is produced by the bayesAB package.

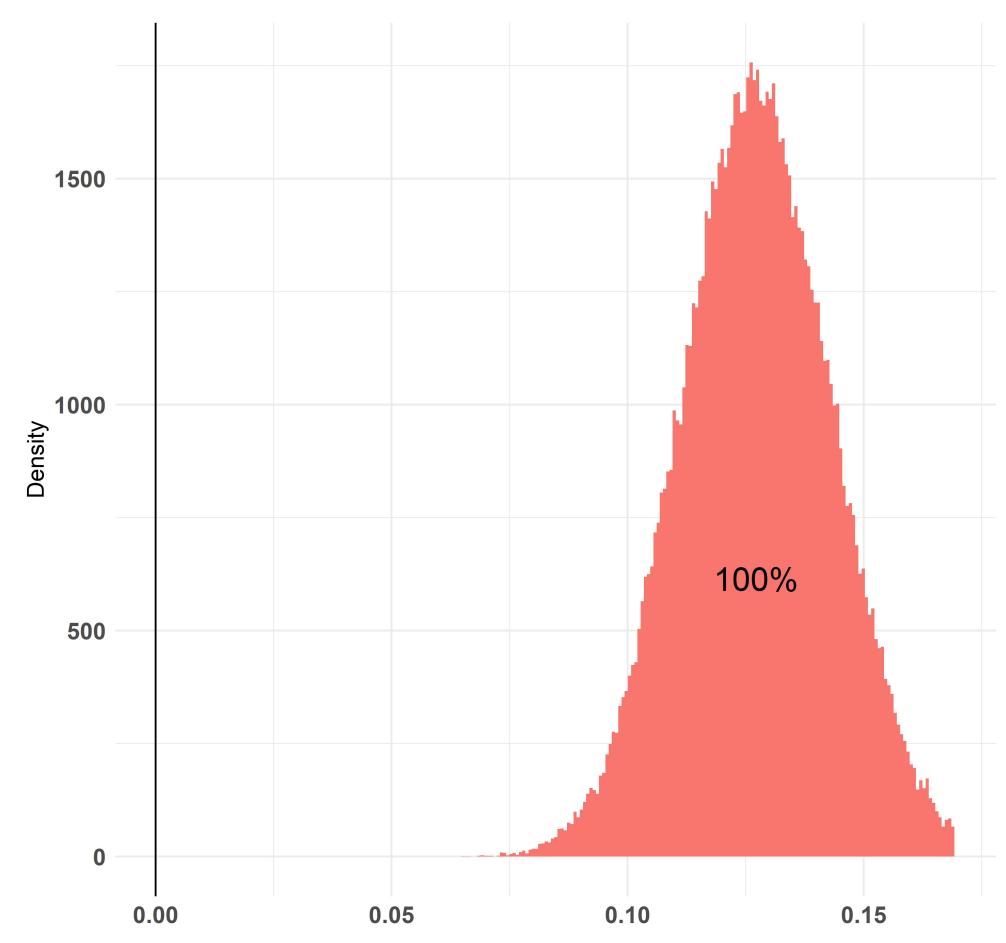

distribution of the difference $\delta$. The distribution is very narrow and it peaks at around 0.1 which means that players in version B played more non-crown games as compared to players in version $\mathrm{A}$.

Figure 16 plots the sequential analysis of the posterior mean of the difference between $\theta_{A}^{\text {non-crown }}$ and $\theta_{B}^{\text {non-crown }}$ as well as the $95 \%$ highest density interval (HDI) of the difference. After some initial fluctuation, the posterior mean difference between $\theta_{A}^{\text {non-crown }}$ and $\theta_{B}^{\text {non-crown }}$ settles at $\sim 0.1$ while the HDI becomes more narrow with increasing sample size. The range of likely values for $\delta$ eventually spans from approximately 0.075 to 0.125 . 


\section{Figure 15}

Posterior distribution of the difference $\delta=\theta_{B}^{\text {non-crown }}-\theta_{A}^{\text {non-crown }}$ for the proportion of non-crown games between the two Rekentuin website versions. Children in version $B$ - the modified website version - played more non-crown games as compared to children playing on the website version $A$.

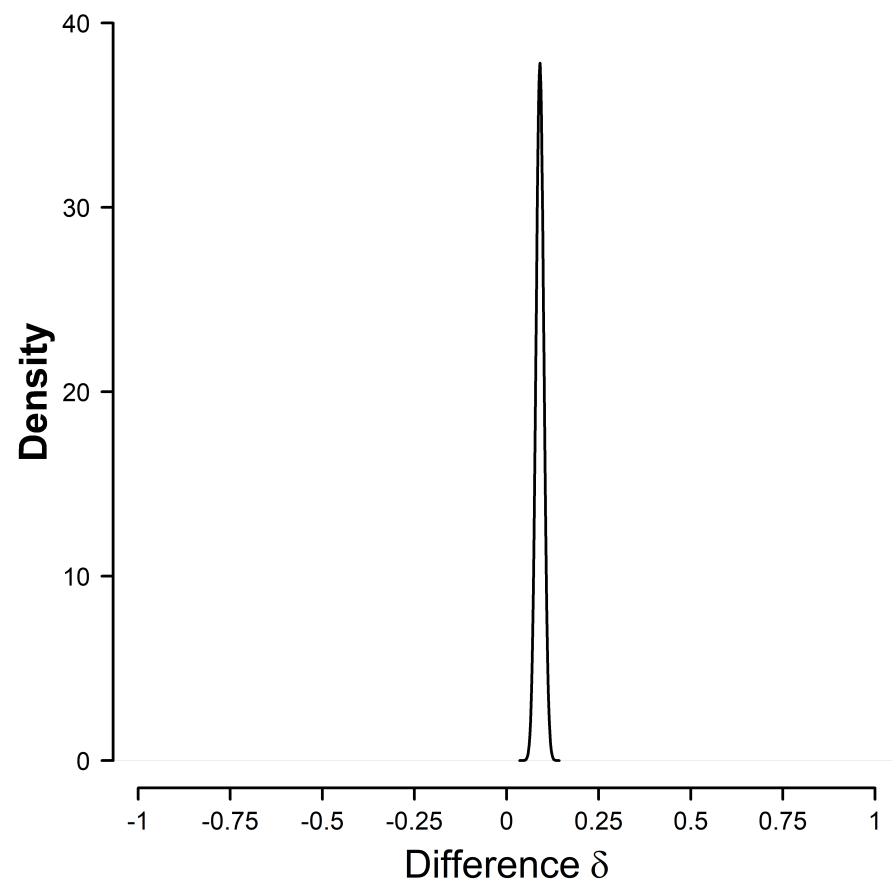

\section{The 'Odds Ratio Approach'}

The observed sample proportions of 0.806 for version $\mathrm{B}$ and 0.715 for version $\mathrm{A}$ suggest that the children in version B played more non-crown games as compared to version $\mathrm{A}$. The Bayes factor $\mathrm{BF}_{+0}$ that assesses the evidence in favor of our hypothesis that the children in version B played more non-crown games equals $7.944 \mathrm{e}+14$. This means that the data are several billion times more likely to occur under the alternative hypothesis $\mathcal{H}_{+}$ than under the null hypothesis $\mathcal{H}_{0}$. In sum, the Bayes factor indicates extreme evidence for the alternative hypothesis (e.g., Jeffreys, 1939; Lee \& Wagenmakers, 2013).

Figure 17 visualizes the dependency of the Bayes factor on the prior distribution on 


\section{Figure 16}

Sequential analysis of the difference between the success probabilities (i.e., $\left.\theta_{B}^{\text {non-crown }}-\theta_{A}^{\text {non-crown }}\right)$ of the two Rekentuin versions. The orange line plots the posterior mean of the absolute risk. The grey area visualizes the width of the highest density interval as a function of sample size $n$.

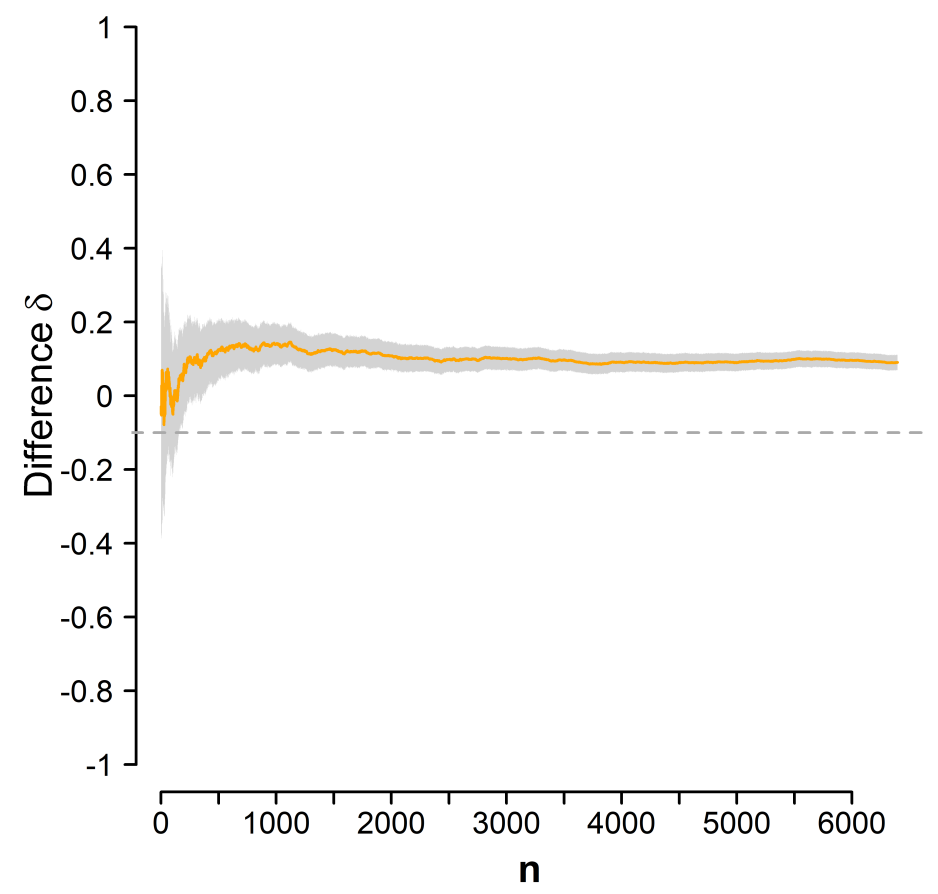

$\psi$ by varying the mean $\mu_{\psi}$ and standard deviation $\sigma_{\psi}$ of the normal prior distribution. From looking at the heatmap we can conclude that the Bayes factor is robust. The data indicate extreme evidence across a range of different values for the prior distribution on $\psi$.

Figure 18 tracks the evidence for either hypothesis in a chronological order. It shows that the evidence for $\mathcal{H}_{+}$is overwhelming from 1000 observations on. The posterior probabilities of the hypotheses are also shown as a probability wheel on the top of Figure 18. The green area visualizes the posterior probability of the alternative hypothesis. The grey area visualizes the posterior probability of the null hypothesis. The data have increased the plausibility of $\mathcal{H}_{+}$from 0.5 to 1 while the posterior plausibility of the null 


\section{Figure 17}

Bayes factor robustness plot for the Rekentuin data.

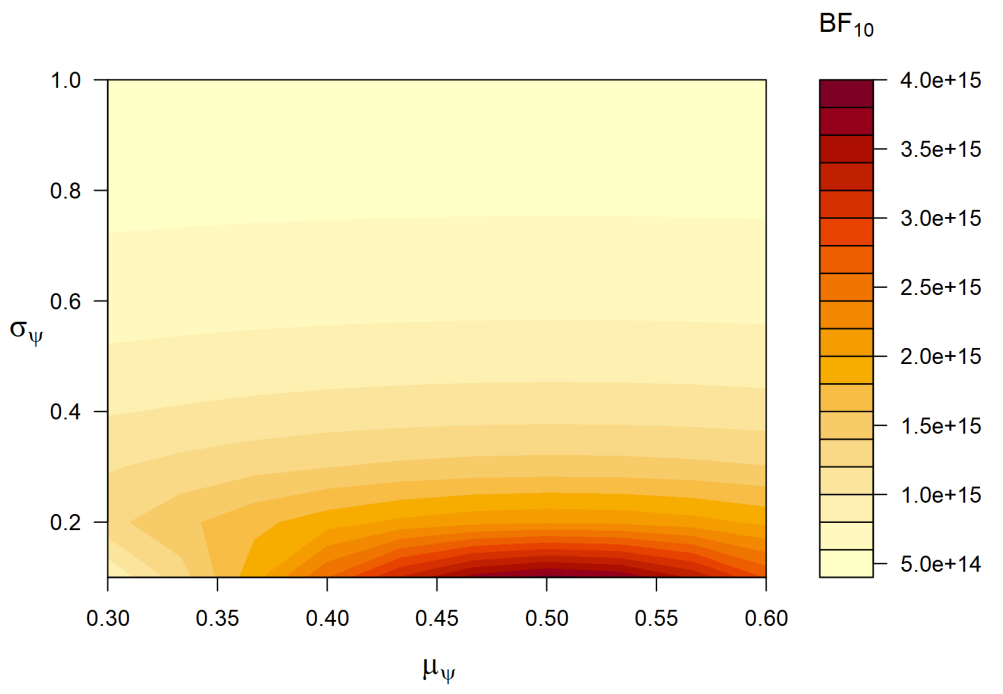

hypothesis $\mathcal{H}_{0}$ has correspondingly decreased from 0.5 to 0.000 .

In sum, there is extreme evidence in favor of the alternative hypothesis. To complete the picture, we quantified the difference between the two Rekentuin versions by estimating the size of the $\log$ odds ratio. Figure 19 shows the prior and posterior distribution for the $\log$ odds ratio under the two-sided model $\mathcal{H}_{1}$. The dotted line displays the prior distribution and the solid line displays the posterior distribution (with $95 \%$ central credible interval). The plot indicates that, given that the log odds ratio is not exactly zero, it is $95 \%$ probable to lie between 0.386 and 0.622 , where the posterior median is 0.504 .

It is important to remember, however, that these results only show because we reversed the assignment of the conditions in the data set. It remains open to investigate why the original data set has such an unexpected pattern. We think that there are two possibilities. As mentioned before the data could have been coded incorrectly; either the conditions or the dependent variable could have been switched. At the same time, we should not ignore the possibility that there are no errors in the data and that this is indeed the true nature of the data. It might be that the children actually enjoyed playing their 


\section{Figure 18}

The flow of posterior probability for $\mathcal{H}_{0}$ and $\mathcal{H}_{+}$as a function of the number of observations across both Rekentuin versions. The prior and posterior probabilities of the hypotheses are displayed on top.

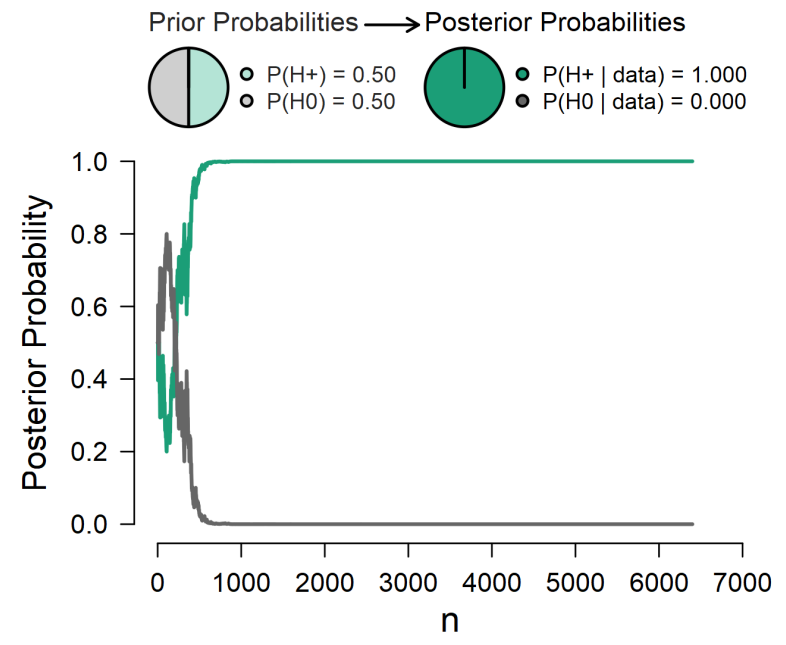

favorite game without experiencing pressure (i.e., having to earn as much coins as possible).

\section{Additional Analyses}

We ran two independent Bayesian $t$-tests on the data set. The first $t$-test examined whether the children reallocated their time amongst the games ${ }^{6}$ Specifically, we compared the proportion of playtime that the children spent on playing crown games across the two versions. We hypothesized that the manipulation in version $\mathrm{B}$ would lead to a decrease in the amount of playtime spent on crown games per child. This analysis allows us to compare the A/B test analysis with an analysis of the same question that uses a different dependent variable and a different statistical procedure. The independent Bayesian $t$-test

\footnotetext{
${ }^{6}$ The time variable in the Rekentuin data set is only an approximation of the children's playing time. We calculated the playing time by subtracting the starting time for consecutive games. Calculated playing times above 600 seconds were changed to NA because we assumed that in that case the children had left the game to return another day.
} 


\section{Figure 19}

Prior and posterior distribution of the log odds ratio under $\mathcal{H}_{1}$ for the Rekentuin data set. The median and the $95 \%$ credible interval of the posterior density for the Rekentuin data are shown in the top right corner.

0.504

$95 \% \mathrm{Cl}:[0.386,0.622]$

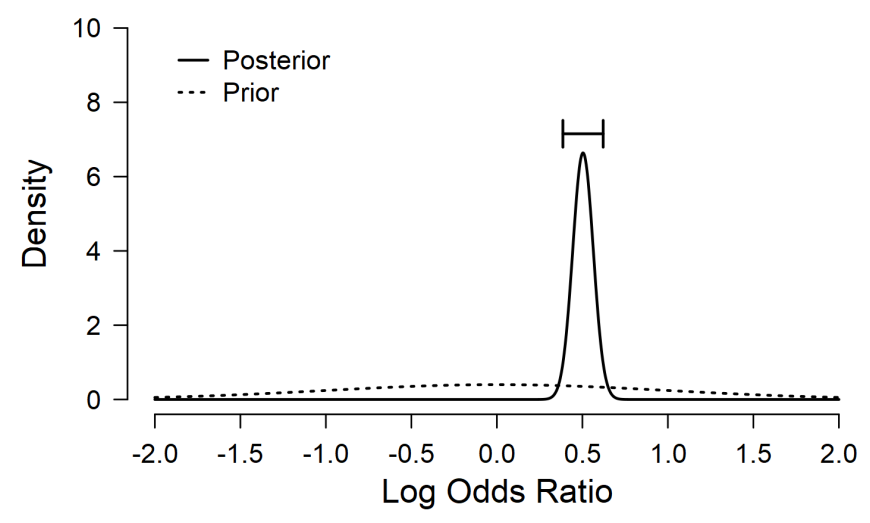

comes to a similar conclusion as the A/B test analysis: The children in version B spent on average a smaller proportion of their playing time on crown games as compared to the children in version A (see Table 2). The data are $2.326 \mathrm{e}+11$ times more likely under the alternative hypothesis $\mathcal{H}_{-}$. A Bayes factor of this size indicates extreme evidence (Lee \& Wagenmakers, 2013). Figure 20 shows that the posterior distribution of the effect size under $\mathcal{H}_{1}$ is concentrated near zero but has all mass below zero. The posterior median is -0.191 and the $95 \%$ CI ranges from -0.240 to -0.142 . We can conclude that the children in version B - the modified Rekentuin version - spent less of their time on Rekentuin playing crown games as compared to the children in version $\mathrm{A}$ - the original Rekentuin version.

The second $t$-test examined whether the reallocation of time was accompanied by a reduction of overall playing time. We hypothesized that the implemented manipulation may have had an unintended and undesirable effect: the children may have reallocated their time from crown games to non-crown games, but, as a consequence, they did not 


\section{Table 2}

Descriptives of the proportion of playtime that was spent on crown games and the summed up playing time (in seconds) in the two Rekentuin versions.

\begin{tabular}{lrrrrrrrr}
\hline & & & & & & & \multicolumn{2}{c}{$95 \%$ Credible Interval } \\
\cline { 9 - 10 } & Version & N & Mean & SD & SE & Lower & Upper \\
\hline \multirow{2}{*}{ proportion crown games } & A & 3177 & 0.290 & 0.284 & 0.005 & 0.280 & 0.300 \\
& B & 3217 & 0.239 & 0.247 & 0.004 & 0.231 & 0.248 \\
\hline total playtime & A & 3177 & 3990.677 & 5191.630 & 92.108 & 3810.080 & 4171.273 \\
& B & 3217 & 3763.501 & 5453.636 & 96.153 & 3574.974 & 3952.027 \\
\hline
\end{tabular}

enjoy playing on the website as much as before and therefore spent less time on Rekentuin overall. To test this conjecture we compared how much time (in seconds) the children spent on Rekentuin altogether, i.e., summed up playtime duration, across the two versions. Table 2 lists the descriptive statistics for this test. The Bayes factor $B F_{0-}$ equals $\sim 4$; meaning that there is moderate evidence for the null hypothesis (Lee \& Wagenmakers, 2013). We can see that the effect size is narrowly concentrated at zero in Figure 21. All told, the results of this $t$-test present moderate evidence for the claim that the manipulation in version B did not lead to a reduction in overall playing time.

In sum, there is strong evidence that removing the coins from crown games led children in version B to spent less time on crown games. Fortunately, this change in their playing behavior was not accompanied by a reduction in overall playing time. Note however that this pattern also only showed because we changed the allocation of the conditions.

\section{Example 2: The Fictional Webshop}

The Rekentuin data is not the most representative example of an A/B test. That is because the manipulation that was implemented on the website targeted the children's underlying motivation to play the games. Common A/B tests for web development 


\section{Figure 20}

Prior and posterior distribution of the effect size for the proportion of playtime spent on crown games under $\mathcal{H}_{1}$ for the Rekentuin data. The median and the $95 \%$ credible interval of the posterior density are shown in the top right corner.

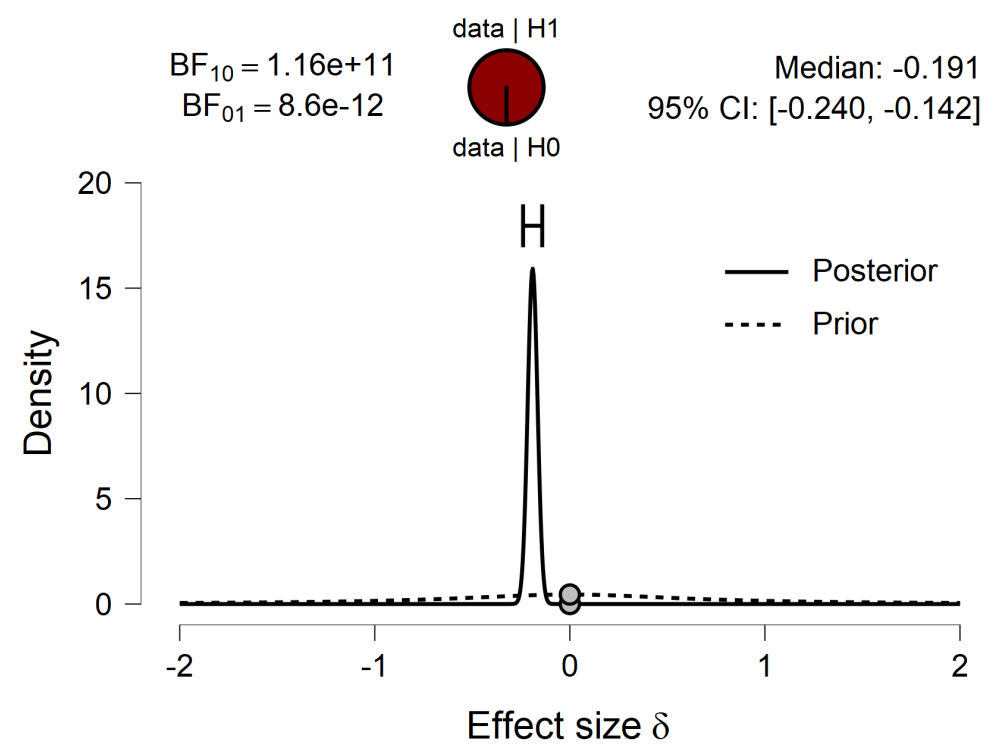

purposes implement much subtler manipulations. In this section we describe an $\mathrm{A} / \mathrm{B}$ test analysis with a more customary data set.

Consider the following fictitious scenario: an online marketing team wants to improve the click rate on a call-to-action button on their website's landing page. Therefore, they devise an A/B test. Half of the website visitors read "Try out our new product." (further denoted as version A), and the other half reads "Test out our new product." (further denoted as version B) when visiting the website.7 The success of the website versions is measured by the rate at which website visitors click on the call-to-action button.

To demonstrate the analyses we use synthetic data. The $\mathrm{R}$ code for the data creation and the data as well as the analysis files can be found at https://osf.io/anvg2/. Table 3 depicts the number of clicks in either group. The conversion rate in version A

\footnotetext{
7 This example was inspired by a real conversion rate optimization project at https://blog.optimizely.com/2011/06/08/optimizely-increases-homepage-conversion-rate-by-29/
} 


\section{Figure 21}

Prior and posterior distribution of the effect size for the summed up playing time under $\mathcal{H}_{1}$ for the Rekentuin data. The median and the $95 \%$ credible interval of the posterior density are shown in the top right corner.

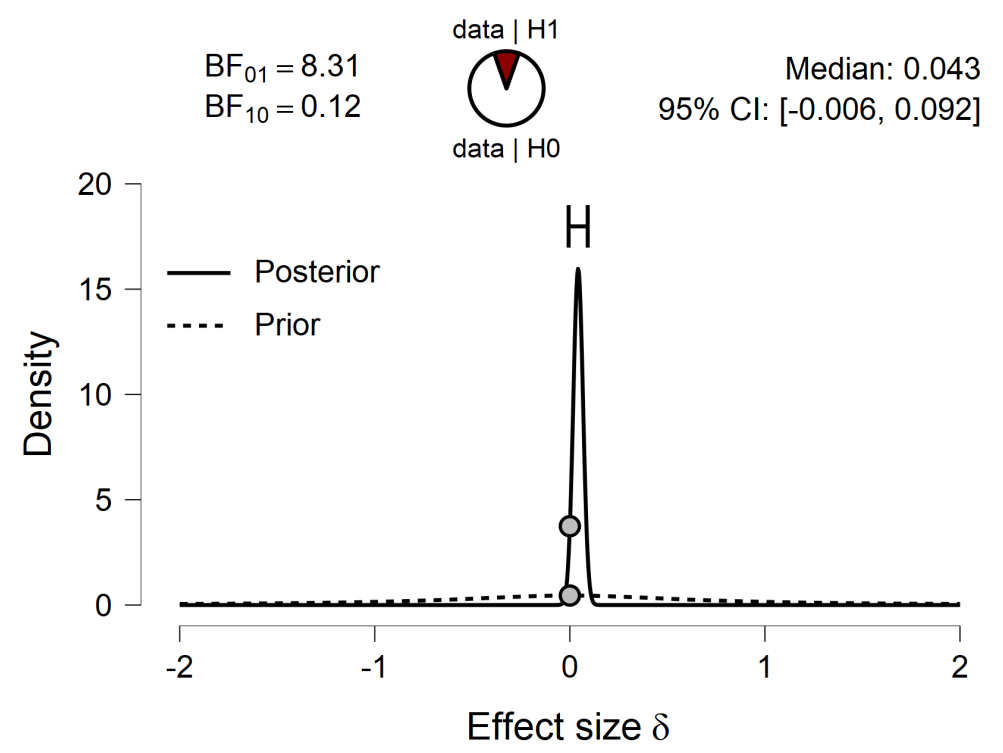

equals 0.113 and 0.128 in version B. The company now wants to determine whether and to what extent the observed sample difference in proportions translates to the population. From a Bayesian perspective, the company wants to assess the evidence in the data for $\mathcal{H}_{+}$ that users in version $\mathrm{B}$ clicked more often as compared to $\mathcal{H}_{0}$ that there is no difference between the two versions.

We will first present the results of the 'common approach' analysis and later contrast them with the results of the 'odds ratio approach.' The dependent variable of both analyses is the click rate, i.e. whether or not a given website visitor clicked on the call-to-action button $(1=$ click, $0=$ no click $)$.

\section{The 'Common Approach'}

We again used the bayes $A B$ package in $R$ to analyse the data according to the 'common approach' (Portman, 2017; R Core Team, 2020). Figure 22a illustrates the two 


\section{Table 3}

Fourfold table indicating the amount of clicks on each webshop version. We used synthetic data for this analysis.

\begin{tabular}{lcc}
\hline & \multicolumn{3}{r}{ Clicked on button } \\
\cline { 2 - 3 } Condition & Yes & No \\
\hline A & 1131 & 8869 \\
B & 1275 & 8725 \\
\hline
\end{tabular}

independent posterior distributions. We can see that version B's posterior distribution for the success probability assigns more mass to higher values of $\theta$. This suggests that the success probability of the modified website version is higher and the alterations had a positive impact on the click rate. Figure 22b depicts the conversion rate uplift. The posterior distribution for the uplift peaks at around 0.125 , indicating that the most likely conversion increase equals $12.5 \%$. Also, most posterior mass (i.e., $99.9 \%$ of the samples) is above zero, indicating that we can be $99.9 \%$ certain that version B is better than version A.

The analytically calculated posterior probability of the event $\theta_{B}>\theta_{A}$ amounts to 0.999 (Schmidt \& Mørup, 2019). Figure 23 shows the posterior difference distribution $\delta$. The distribution peaks at about 0.02. A difference of this size is quite large for a conversion rate optimization endeavor. We calculated $\delta$ for this data set with the approach described in Appendix B.

Figure 24 plots the posterior mean of the difference between $\theta_{A}$ and $\theta_{B}$ as well as the $95 \%$ highest density interval (HDI) of the difference in a sequential manner. With increasing sample size, the HDI becomes more narrow. This indicates that the range of likely values for $\delta$ becomes smaller. We can see in Figure 24b that after some initial fluctuation, the posterior mean difference between the two success probabilities $\theta_{A}$ and $\theta_{B}$ settles between 0 and 0.05 . 


\section{Figure 22}

(a) Independent posterior beta distributions of the success probabilities for version $A$ and $B$. The plot is produced by the bayesAB package with the fictional webshop data. The analysis used two independent beta $(1,1)$ priors.

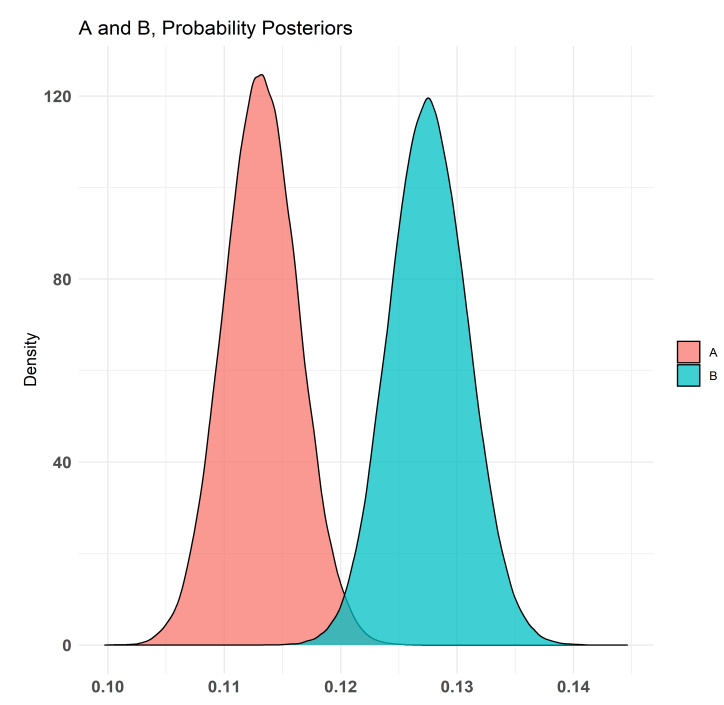

(b) Histogram of the conversion rate uplift from version $A$ to version $B$. The uplift is calculated by dividing the difference in conversion by the conversion in $A$. The plot is produced by the bayesAB package.

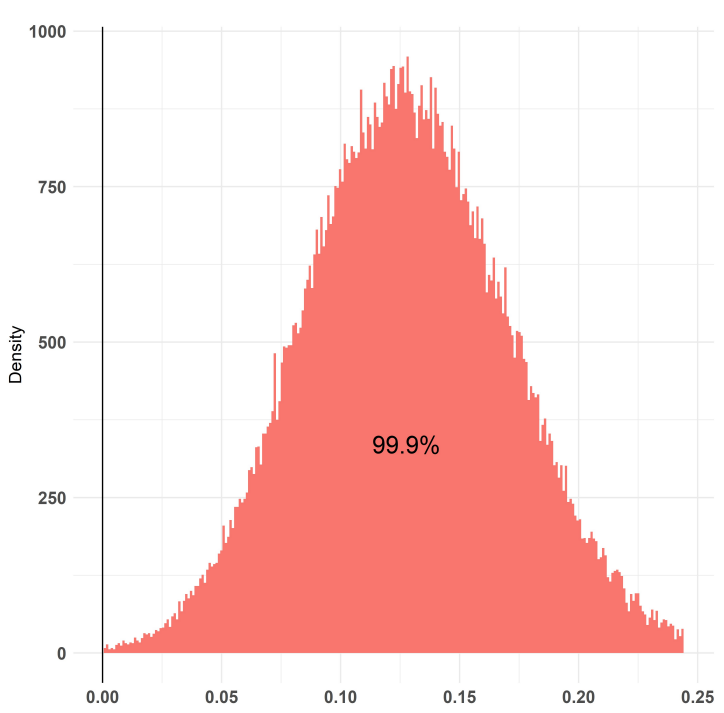

\section{The 'Odds Ratio Approach'}

Before the data can be analysed according to the 'odds ratio approach', a prior distribution for the log odds ratio has to be specified. For this purpose it is important to note that the subtle manipulations of common A/B tests lead to very small effect sizes. The effect size of website changes, i.e., the difference in conversion rates between the baseline version and its modification, is typically as small as $0.5 \%$ and less (Berman et al., 2018). This means that the analysis of such data requires an exceptionally narrow prior distribution that peaks at a value close to 0 as the shape of the prior distribution has to map the relative plausibility of parameter values.

For the present example we can compare the influence of different prior distributions on the analysis. Suppose that the online marketing team specifies three prior 


\section{Figure 23}

The difference $\delta$ (i.e., $\theta_{B}-\theta_{A}$ ) between the two independent beta distributions for the fictitious webshop example.

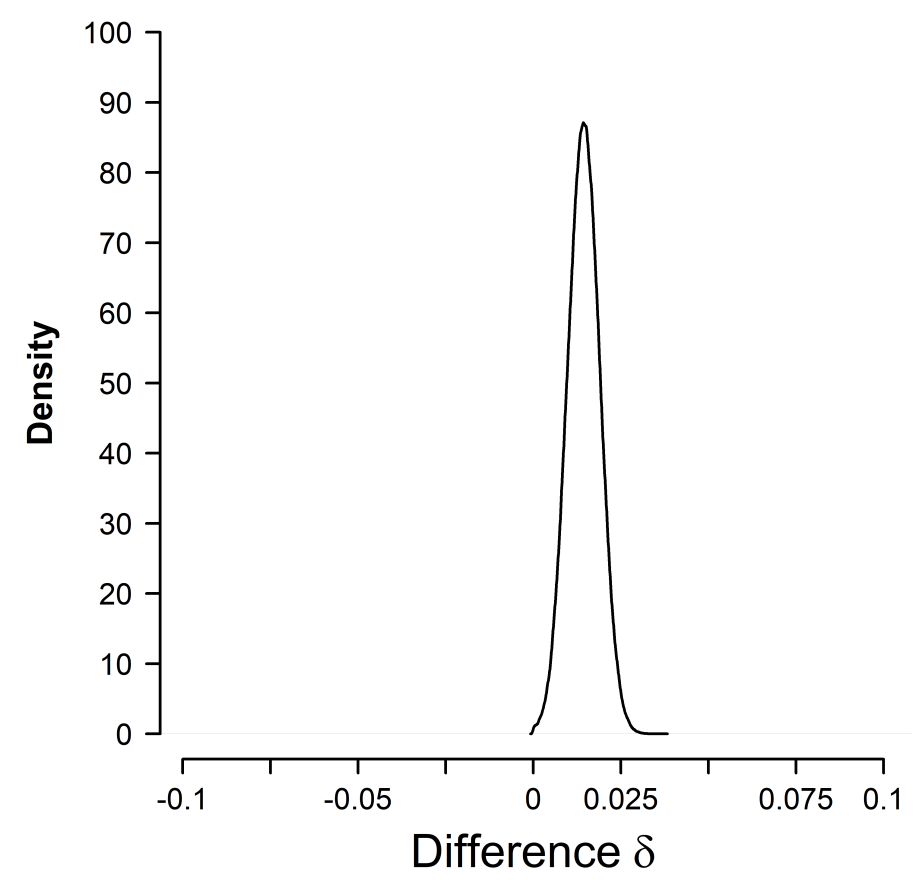

distributions: Most team members are inexperienced with conversion rate optimization. Therefore they are indifferent about plausible values of the log odds ratio and prefer to use the commonly employed uninformed normal prior. The prior is however truncated as they expect a positive effect (see Figure 25a). The team members with prior knowledge on A/B testing specify more narrow distributions that center around a value above zero. One team member is quite optimistic about the conversion rate increase in version B. He thinks that the odds ratio will be around 1.2 (see Figure 25c). Another team member thinks this is too extreme. Her more conservative estimate is an odds ratio of 1.05 (see Figure 25b). In sum, the data will be analysed with three priors: the indifferent prior with $\mu_{\psi}=0$ and $\sigma_{\psi}=1$, the optimistic prior with $\mu_{\psi}=0.18$ and $\sigma_{\psi}=0.005$, the conservative prior with $\mu_{\psi}=0.05$ and $\sigma_{\psi}=0.03$. 


\section{Figure 24}

Sequential analysis of the difference $\theta_{B}-\theta_{A}$ of the two fictitious webshop versions. The orange line plots the posterior mean of the absolute risk. The grey area visualizes the width of the highest density interval as a function of sample size $n$.

(a) Sequential analysis of the difference. For a close-up see Figure 246

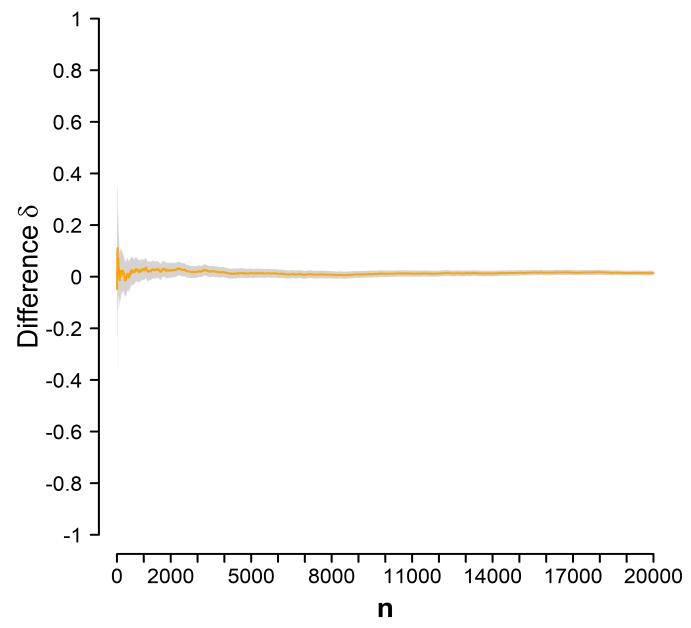

(b) This plot shows the same as the plot on the left, but the limits of the y-axis are more narrow.

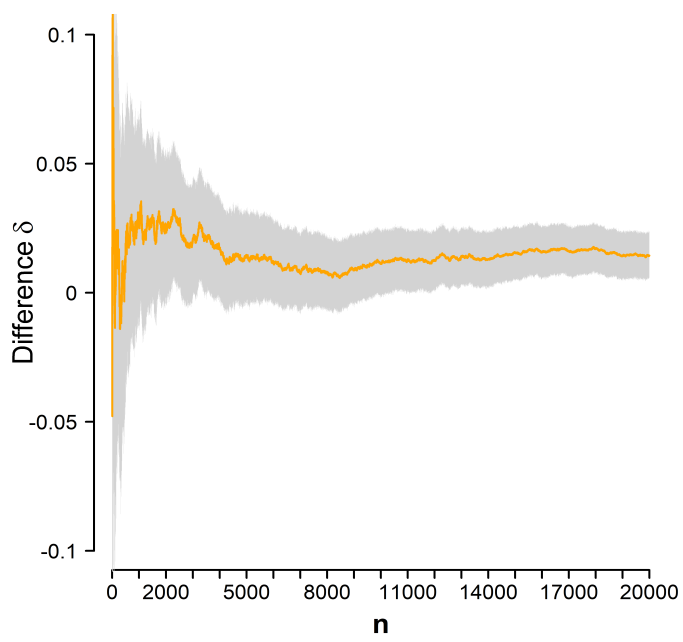

We used the abtest package (Gronau, 2019) in $\mathrm{R}$ ( $\mathrm{R}$ Core Team, 2020) and JASP for the present analysis. Before estimating the effect, we evaluate the evidence presented by the data that there is indeed a difference between version A and B. Overall the data support the hypothesis that users in version B clicked more often as compared to users in version A. The data are approximately 11 times more likely under the alternative hypothesis $\mathcal{H}_{+}$than under the null hypothesis $\mathcal{H}_{0}$ for the indifferent prior. They are $\sim 27$ times more likely under the alternative hypothesis for the conservative prior, and $\sim 80$ times more likely under $\mathcal{H}_{+}$for the optimistic prior. The influence of the prior on the Bayes factor can be explored more broadly with the Bayes factor robustness plot. Figure 26 plots the corresponding heatmap. Varying the standard deviation $\sigma_{\psi}$ of the prior distribution on $\psi$ shows that the $\mathrm{BF}_{10}$ changes from very strong to moderate evidence. 
This illustrates the risk of applying an uninformed prior distribution that assigns probability to unreasonable parameter values. Figure 26 shows that the evidence is robust if the prior distribution utilizes reasonable $\mu_{\psi}$ and $\sigma_{\psi}$ values. Hence, it should be reflected in the prior distribution if an effect can be anticipated to be very small.

Figure 27 plots the probability wheels of the hypotheses. The most left plot illustrates the probabilities of the hypotheses for the indifferent (standard normal) prior. Figure 27b shows the change of probability for the conservative prior. The change from prior to posterior is largest for the optimistic prior which is displayed in Figure $27 \mathrm{c}$. For all three prior distributions the alternative hypothesis $\mathcal{H}_{+}$has a much higher posterior probability $(>0.9)$ as compared to the null hypothesis.

Figure 28 plots the evidential trajectory for the three prior distributions. The evidence fluctuates a good deal for the first 10,000 observations for the analysis with the standard normal prior - in phases the data provide more evidence for $\mathcal{H}_{+}$and then again for $\mathcal{H}_{0}$. After a tie at 10,000 observation $\mathcal{H}_{+}$triumphs and the posterior probability of $\mathcal{H}_{+}$

Figure 25

(a) Truncated standard normal

(b) The conservative prior. $\quad$ (c) The optimistic prior. The prior. The odds ratio is assumed to be odds ratio is assumed to be $\sim 1.05$ $\sim 1.2$
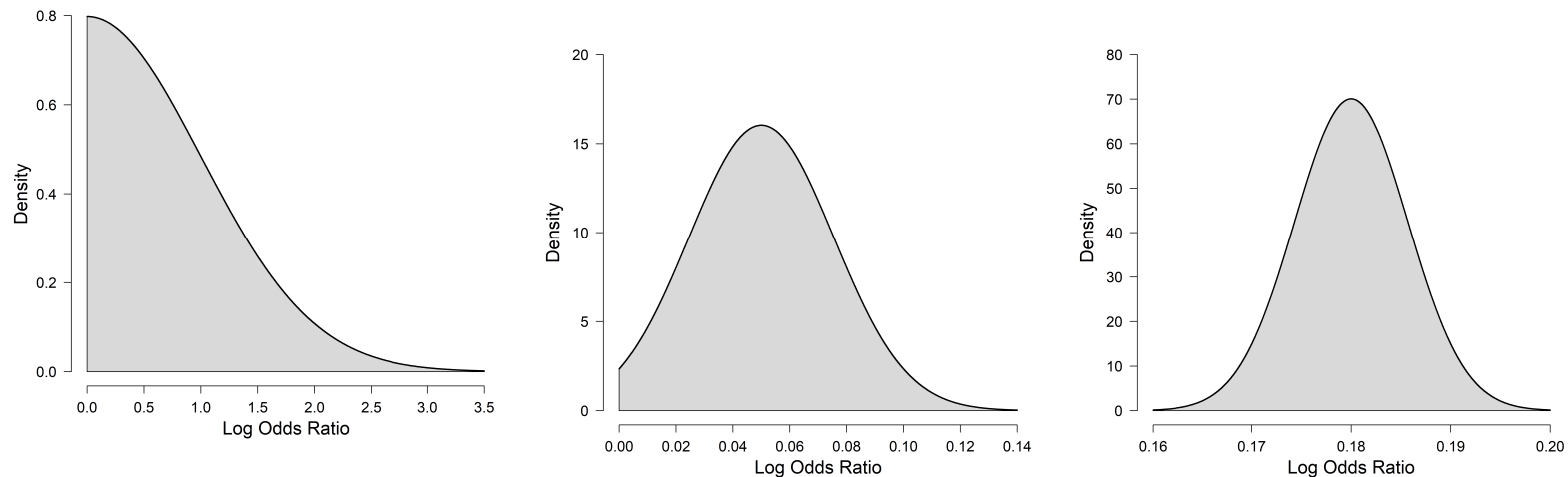


\section{Figure 26}

Bayes factor robustness plot for the fictitious webshop data. Overall, the conclusion that there is strong evidence for $\mathcal{H}_{1}$ over $\mathcal{H}_{0}$ seems robust across a range of reasonable values for $\mu_{\psi}$ and $\sigma_{\psi}$. The evidence is not compelling if the analysis utilizes a very wide prior (i.e., for high values for $\sigma_{\psi}$ )

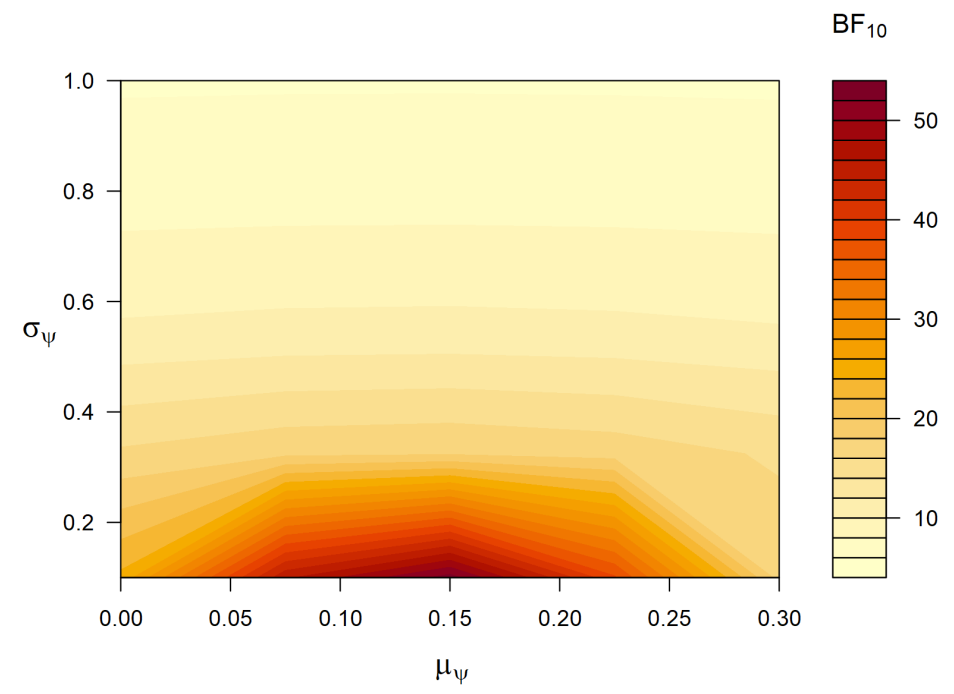

rises substantially while the posterior probability of $\mathcal{H}_{0}$ decreases accordingly. By contrast, the sequential analysis with the conservative prior is unambiguous (see Figure 28b). The posterior probability of $\mathcal{H}_{+}$dominates throughout all observations. The analysis with the optimistic prior produces a similar picture as the analysis with the standard normal prior (see Figure 28c). The probability of $\mathcal{H}_{+}$increases sharply after some initial fluctuation and a tie at about 10,000 observations.

In sum, the data present strong to very strong evidence for the claim that the conversion rate is higher in version B (Lee \& Wagenmakers, 2013). Knowing this, the online marketing team wants to assess the size of the effect. Figure 29 displays the prior and posterior distribution for the log odds ratio using the three different priors. The prior distribution is plotted as a dotted line and the posterior distribution as a solid line (with 95\% central credible interval). Under the assumption that the effect is non-zero, Figure 29a 


\section{Figure 27}

Probabilities of the hypotheses after taking the fictitious webshop data into account. The green area visualizes the posterior probability of the alternative hypothesis. The grey area visualizes the posterior probability of the null hypothesis. Both hypotheses were assigned a probability of 0.5 before testing.

(a) Posterior probabilities of

the hypotheses under the

standard normal prior.

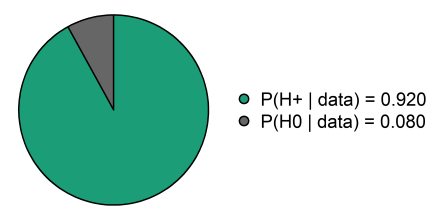

(b) Posterior probabilities of

the hypotheses under the

conservative prior.

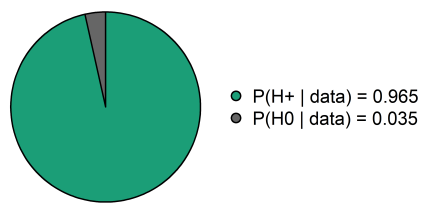

(c) Posterior probabilities of

the hypotheses under the

optimistic prior.

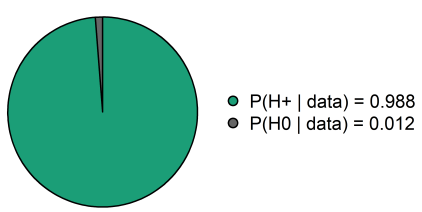

indicates that the posterior median of the log odds ratio is 0.135 with a $95 \%$ credible interval (CI) ranging from 0.050 to 0.220 when using the standard normal prior. The middle panel of Figure 29 displays the posterior distribution for the log odds ratio when using the conservative prior. The posterior distribution indicates that the effect is slightly bigger than expected under this prior. The distribution peaks at 0.072 and the $95 \%$ CI ranges from 0.029 to 0.116 . Figure $29 \mathrm{c}$ indicates that given the log odds ratio is not exactly zero, the posterior median is 0.179 when using the optimistic prior. The $95 \%$ CI ranges from 0.168 to 0.190 . In fact, the optimistic prior is very similar to its corresponding posterior distribution of the log odds ratio.

Lastly, to compare the predictive performance of the three different models, the company calculates Bayes factors with the resulting marginal likelihoods. The comparison clarifies that the three models are very similar. The data are 1.5 times more likely under the conservative model than under the indifferent model. They are 4.5 times more likely 
under the optimistic than under the indifferent model. And the data are 2.9 times more likely under the optimistic than under the conservative model. In verbal terms, these Bayes factors correspond to 'anecdotal' or 'moderate' evidence (Lee \& Wagenmakers, 2013).

\section{Concluding Comments}

Data are an important resource for modern companies. The race is on in any market where lots of data is available and whoever learns fastest from their data will triumph (Domingos, 2015). However too many decision are made with a limited understanding of the statistical analysis in use. By knowing what a particular test does, businesses can be sure to understand the information they receive from their data and eventually to optimize what they care about.

With lots of data to go with it, Bayesian A/B testing is a tool that transforms data into knowledge. We showed that the Bayesian approach to A/B testing allows applicants to answer a wide array of questions that are relevant in the web development domain. With our demonstrations we hope to inspire companies to use the Bayesian approach and

\section{Figure 28}

The flow of posterior probability for $\mathcal{H}_{0}$ and $\mathcal{H}_{+}$as a function of the number of observations across both fictitious website versions.

(a) Sequential analysis with the (b) Sequential analysis with the (c) Sequential analysis with the standard normal prior. conservative prior.

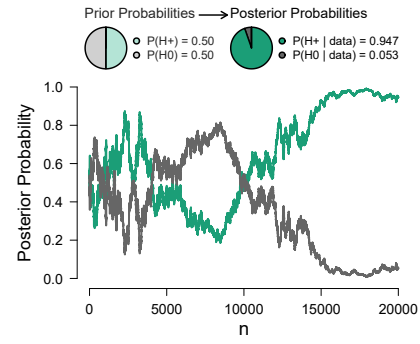

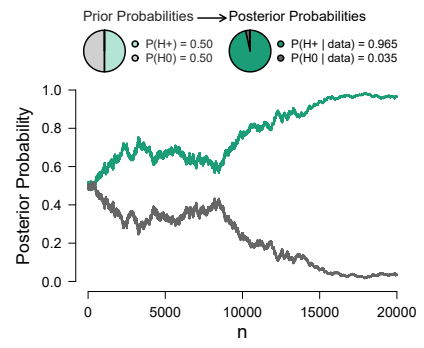
optimistic prior.

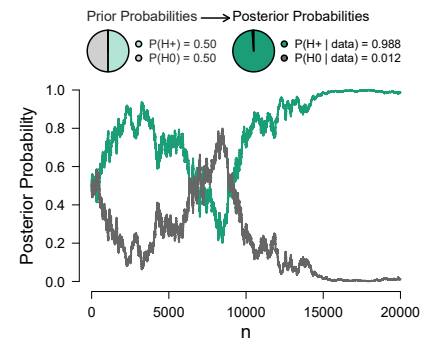




\section{Figure 29}

(a) Truncated standard normal prior.
(b) The conservative prior.
(c) The optimistic prior.
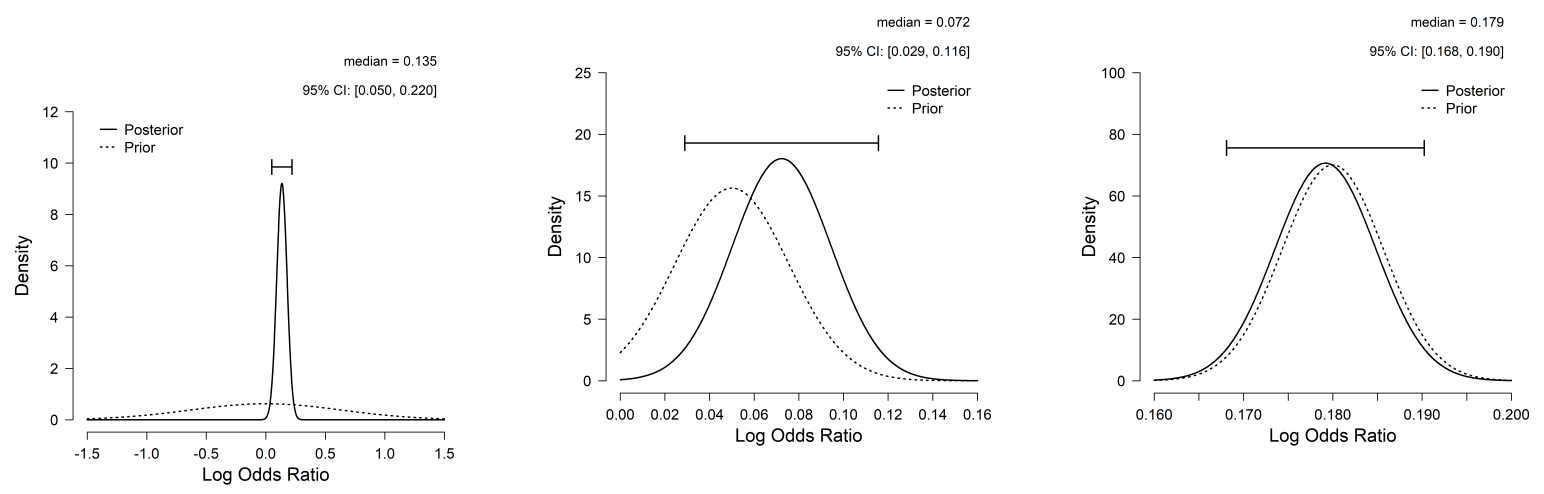

to inform them on how to use it to their competitive advantage.

Especially the 'odds ratio approach' fulfills the general desiderata that an A/B test should meet: The approach allows practitioners to obtain evidence in favor of the null hypothesis, to monitor the evidence as the data accumulate, and to take prior/expert knowledge into account. The benefits of a test that fulfills these three desiderata are manifold. For a start it affords companies with economicalness. That is because the sequential analysis prevents a waste of resources - the testing can be stopped as soon as the evidence is compelling. This also means that the company can make decisions much quicker since they do not have to stick to some predefined sampling plan. Another advantage is that the 'odds ratio approach' allows practitioners to test the influence of different priors and examine how well those predict the data. In the long run, companies can build a database of prior distributions where they incorporate what was learned from previous data; paying tribute to Lindley's axiom "today's posterior is tomorrow's prior" (Lindley, 1972). Lastly, being able to obtain evidence in favor of the null hypothesis enables practitioners to ascertain that there is truly no difference between two website 
versions. This comes in handy whenever practitioners want to ensure that, for example, technical changes on a website did not hamper website visitor's experience. Moreover is it valuable to know if a certain implementation does not have the desired effect. Knowing for sure that there is no effect allows practitioners to stop the ongoing experiment in good conscience and thereby save money, time and other resources. 


\section{References}

Altman, D. G., \& Bland, J. M. (1995). Statistics notes: Absence of evidence is not evidence of absence. BMJ, 311(7003), 485.

Berman, R., Pekelis, L., Scott, A., \& Van den Bulte, C. (2018). P-hacking and false discovery in A/B testing. https://ssrn.com/abstract=3204791

Bondarenko, Y. S., \& Kravchenko, S. (2019). Bayesian approach to landing page testing. Problems of Applied Mathematics and Mathematical Modeling.

Cameron, J., Banko, K. M., \& Pierce, W. D. (2001). Pervasive negative effects of rewards on intrinsic motivation: The myth continues. The Behavior Analyst, 24(1), 1-44.

Deng, A. (2015). Objective bayesian two sample hypothesis testing for online controlled experiments, In $W W W^{\prime} 15$ companion: Proceedings of the 24th international conference on world wide web.

Deng, A., Lu, J., \& Chen, S. (2016). Continuous monitoring of A/B tests without pain: Optional stopping in bayesian testing, In 2016 IEEE international conference on data science and advanced analytics (DSAA). IEEE.

Domingos, P. (2015). The master algorithm: How the quest for the ultimate learning machine will remake our world. Basic Books.

Gelman, A., Carlin, J. B., Stern, H. S., Dunson, D. B., Vehtari, A., \& Rubin, D. B. (2013). Bayesian data analysis. CRC press.

Goodson, M. (2014). Most winning A/B test results are illusory. Whitepaper Qubit, https://tinyurl.com/y9g3m9bq.

Gronau, Q. F. (2019). Abtest: Bayesian A/B testing. https://CRAN.R-project.org/package=abtest

Gronau, Q. F., Raj K N, A., \& Wagenmakers, E.-J. (2019). Informed Bayesian inference for the A/B test, arXiv 1905.02068.

Gudigantala, N., Bicen, P. Et al. (2016). An examination of antecedents of conversion rates of e-commerce retailers. Management Research Review, 39(1), 82-114. 
Howard, J. (1998). The $2 \times 2$ table: A discussion from a Bayesian viewpoint. Statistical Science, 351-367.

JASP Team. (2020). JASP (Version 0.13)[Computer software]. https://jasp-stats.org/

Jeffreys, H. (1939). Theory of probability (1st ed.). Oxford, UK, Oxford University Press.

Jeffreys, H. (1961). Theory of probability (3rd ed.). Oxford, UK, Oxford University Press.

Jennison, C., Turnbull, B. W. Et al. (1990). Statistical approaches to interim monitoring of medical trials: A review and commentary. Statistical Science, 5(3), 299-317.

Johnson, G., Lewis, R. A., \& Nubbemeyer, E. (2017). The online display ad effectiveness funnel \& carryover: Lessons from 432 field experiments. https://papers.ssrn.com/sol3/papers.cfm?abstract_id=2701578

Kamalbasha, S., \& Eugster, M. J. A. (2020). Bayesian A/B testing for business decisions, arXiv 2003.02769.

Kass, R. E., \& Raftery, A. E. (1995). Bayes factors. Journal of the American Statistical Association, 90, 773-795.

Kass, R. E., \& Vaidyanathan, S. K. (1992). Approximate Bayes factors and orthogonal parameters, with application to testing equality of two binomial proportions. Journal of the Royal Statistical Society, Series B, 54, 129-144.

Kruschke, J. K. (2013). Bayesian estimation supersedes the $t$ test. Journal of Experimental Psychology: General, 142(2), 573-603.

Lee, M. D., \& Wagenmakers, E.-J. (2013). Bayesian cognitive modeling: A practical course. Cambridge University Press.

Lindley, D. V. (1972). Bayesian statistics, a review. Philadelphia (PA), SIAM.

Lindley, D. V. (1993). The analysis of experimental data: The appreciation of tea and wine. Teaching statistics, 15(1), 22-25.

Little, R. J. (1989). Testing the equality of two independent binomial proportions. The American Statistician, 43(4), 283-288. 
McFarland, C. (2012). Experiment!: Website conversion rate optimization with $A / B$ and multivariate testing. Berkeley, CA, New Riders.

Munafò, M. R., Nosek, B. A., Bishop, D. V., Button, K. S., Chambers, C. D., Du Sert, N. P., Simonsohn, U., Wagenmakers, E.-J., Ware, J. J., \& Ioannidis, J. P. (2017). A manifesto for reproducible science. Nature Human Behaviour, 1(1), 1-9.

Patel, N. (2018). What is a good conversion rate? The answer might surprise you. https://www.crazyegg.com/blog/what-is-good-conversion-rate/

Pham-Gia, T., Turkkan, N., \& Eng, P. (1993). Bayesian analysis of the difference of two proportions. Communications in Statistics-Theory and Methods, 22(6), 1755-1771.

Portman, F. (2017). Bayesab: Fast Bayesian methods for A/B testing. https://cran.r-project.org/web/packages/bayesAB/index.html

R Core Team. (2020). R: A language and environment for statistical computing. $\mathrm{R}$ Foundation for Statistical Computing. Vienna, Austria. https://www.R-project.org/

Saleh, K., \& Shukairy, A. (2010). Conversion optimization: The art and science of converting prospects to customers. Sebastopol (CA), O’Reilly Media, Inc.

Schmidt, M. N., \& Mørup, M. (2019). Efficient computation for Bayesian comparison of two proportions. Statistics $\&$ Probability Letters, 145, 57-62.

Singh, V. (2019). What is Bayesian statistics? VWO Knowledge Base. https://help.vwo.com/hc/en-us/articles/360033991193-What-Is-Bayesian-Statistics-

Stucchio, C. (2015). Bayesian A/B testing at VWO. Whitepaper, Visual Website Optimizer. Tang, S.-H., \& Hall, V. C. (1995). The overjustification effect: A meta-analysis. Applied Cognitive Psychology, 9(5), 365-404.

van Doorn, J., Matzke, D., \& Wagenmakers, E.-J. (2020). An in-class demonstration of Bayesian inference. Psychology Learning \& Teaching, 19(1), 36-45.

Wagenmakers, E.-J. (2007). A practical solution to the pervasive problems of p values. Psychonomic Bulletin \& Review, 14(5), 779-804. 
Wagenmakers, E.-J., Marsman, M., Jamil, T., Ly, A., Verhagen, J., Love, J., Selker, R., Gronau, Q. F., Šmıra, M., Epskamp, S., Et al. (2018). Bayesian inference for psychology. Part I: Theoretical advantages and practical ramifications. Psychonomic Bulletin \& Review, 25(1), 35-57.

Wagenmakers, E.-J., Morey, R. D., \& Lee, M. D. (2016). Bayesian benefits for the pragmatic researcher. Current Directions in Psychological Science, 25(3), 169-176.

Williams, M. N., Bååth, R. A., \& Philipp, M. C. (2017). Using Bayes factors to test hypotheses in developmental research. Research in Human Development, 14 (4), 321-337.

Wrinch, D., \& Jeffreys, H. (1921). On certain fundamental principles of scientific inquiry. Philosophical Magazine, 42, 369-390. 


\section{Appendix A}

\section{Formulae}

Schmidt and Mørup, 2019

Let T denote a $2 \times 2$ contingency table with fixed column total $n_{1}$ and $n_{2}$,

$$
T=\left[\begin{array}{cc}
y 1 & y 2 \\
n 1-y 1 & n 2-y 2
\end{array}\right] .
$$

The posterior probability of the event $P\left(\theta_{1}>\theta_{2} \mid T\right)$ is given by

$$
P\left(\theta_{1}>\theta_{2} \mid T\right)=\frac{Z\left(\alpha_{1}, \beta_{1}, \alpha_{2}, \beta_{2}\right)}{B\left(\alpha_{1}, \beta_{1}\right) B\left(\alpha_{2}, \beta_{2}\right)}
$$

where $B(\alpha, \beta)$ is the Beta function. The normalizing constant $Z$ is given by

$$
Z\left(\alpha_{1}, \beta_{1}, \alpha_{2}, \beta_{2}\right)=\frac{\Gamma\left(\alpha_{1}+\alpha_{2}\right) \Gamma\left(\beta_{1}+\beta_{2}\right)}{\beta_{1} \alpha_{2} \Gamma\left(\alpha_{1}+\beta_{1}+\alpha_{2}+\beta_{2}-1\right)}{ }_{3} F_{2}\left[\begin{array}{c}
1,1-\alpha_{1}, 1-\beta_{2} \\
\beta_{1}+1, \alpha_{2}+1
\end{array} ;\right]
$$

where ${ }_{3} F_{2}$ is the generalized hypergeometric function.

Pham-Gia et al., 1993

Let $p_{i} \sim \operatorname{beta}\left(\alpha_{i}, \beta_{i}\right), i=1,2$, be independent variables. Then $p=p_{1}-p_{2}$ has the following density:

For $0<p \leq 1, f(p)=$

$B\left(\alpha_{2}, \beta_{1}\right) p^{\beta_{1}+\beta_{2}-1}(1-p)^{\alpha_{2}+\beta_{1}-1} F_{1}\left(\beta_{1}, \alpha_{1}+\beta_{1}+\alpha_{2}+\beta_{2}-2,1-\alpha_{1} ; \beta_{1}+\alpha_{2} ;(1-p), 1-p^{2}\right) / A$,

and for $-1 \leq p<0$

$f(p)=B\left(\alpha_{1}, \beta_{2}\right)(-p)^{\beta_{1}+\beta_{2}-1}(1+p)^{\alpha_{1}+\beta_{2}-1} F_{1}\left(\beta_{2}, 1-\alpha_{2}, \alpha_{1}+\alpha_{2}+\beta_{1}+\beta_{2}-2 ; \alpha_{1}+\beta_{2} ; 1-p^{2}, 1+p\right) / A$,

where $A=B\left(\alpha_{1}, \beta_{1}\right) B\left(\alpha_{2}, \beta_{2}\right)$.

Moreover, if $\alpha_{1}+\alpha_{2}>1$ and $\beta_{1}+\beta_{2}>1$ we have:

$$
f(0)=B\left(\alpha_{1}+\alpha_{2}-1, \beta_{1}+\beta_{2}-1\right) / A
$$




\section{Normal Approximation of Beta Difference}

The $\operatorname{Beta}(\alpha, \beta)$ function can be approximated by the Normal distribution when $\alpha$ and $\beta$ are sufficiently large:

$$
\operatorname{Beta}(\alpha, \beta) \hat{=} \operatorname{Normal}\left(\frac{\alpha}{\alpha+\beta}, \sqrt{\frac{\alpha \beta}{(\alpha+\beta)^{2}(\alpha+\beta+1)}}\right)
$$

The distribution of the difference between two normally distributed variables

$$
X \sim N\left(\mu_{X}, \sigma_{X}^{2}\right)
$$

and

$$
Y \sim N\left(\mu_{Y}, \sigma_{Y}^{2}\right)
$$

is given by another Normal distribution

$$
\delta(X-Y) \sim N\left(\mu_{X}-\mu_{Y}, \sigma_{X}^{2}+\sigma_{Y}^{2}\right)
$$




\section{Appendix B}

\section{On Calculating the Difference between Two Independent Beta Distributions}

We considered three ways of inspecting the difference between the two versions:

analytically calculating it, approximating it with a normal distribution, or using the 'odds ratio approach.' We would suggest that the 'odds ratio approach' is most useful whenever practitioners are interested in testing and quantifying the difference between two versions.

However, in practice, the 'odds ratio approach' is virtually never used. It is therefore beneficial to review which of the remaining methods is most useful to quantify this difference. In addition to using the formula by Pham-Gia et al. (1993) or the normal approximation, there is another possibility to calculate $P(\delta>0)$ : inspecting the posterior probability of $\mathcal{H}_{+}$when comparing $\mathcal{H}_{+}$and $\mathcal{H}_{-}$. While this is exactly what the "common approach' does - gauging whether there is either a positive or a negative effect - we do not receive the posterior probability of the hypotheses when using the bayes AB package. This is because of the approach's strong focus on estimation. It is however easy to acquire the probability with the abtest package:

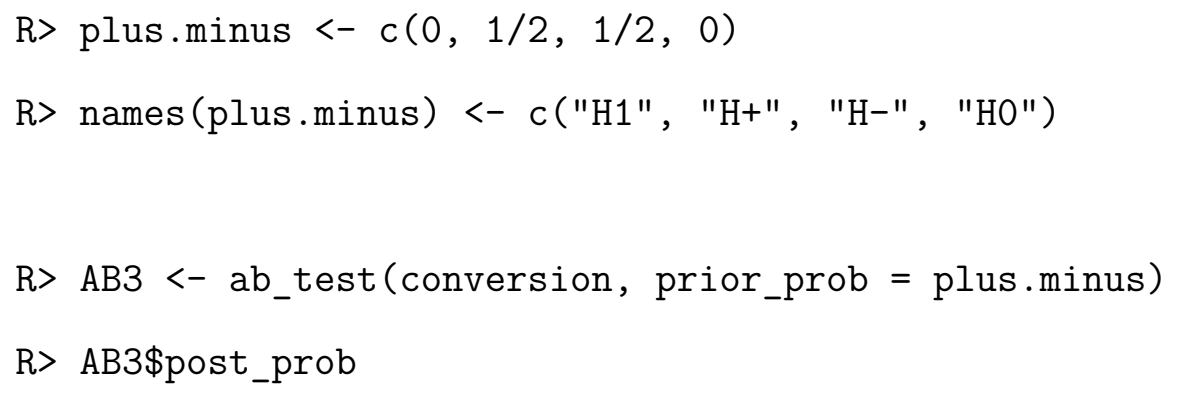

Figure B1a now visualises the sequential analysis of $P(\delta>0)$ for the three different methods. While the analytic calculation of the difference is the most accurate, it fails for large values of $\alpha$ and $\beta$. For the present example, we cannot calculate $P(\delta>0)$ with the exact approach from $n \approx 100$. This is unfortunate as websites are often visited by a large number of people.

As mentioned before, the normal approximation can be used as an alternative to the analytical solution. Specifically, the independent posterior beta distributions can be 


\section{Figure B1}

Sequential analysis of $P(\delta>0)$.

(a) Sequential analysis of the probability that $\theta_{B}$ is larger than $\theta_{A}$ for $N=200$. The striped line visualizes the exact difference. Note that the calculations break down for $n \approx 70$

(b) Sequential analysis of $P(\delta>0)$ for a larger data set of $N=20000$. The calculation of the exact difference quickly breaks down.
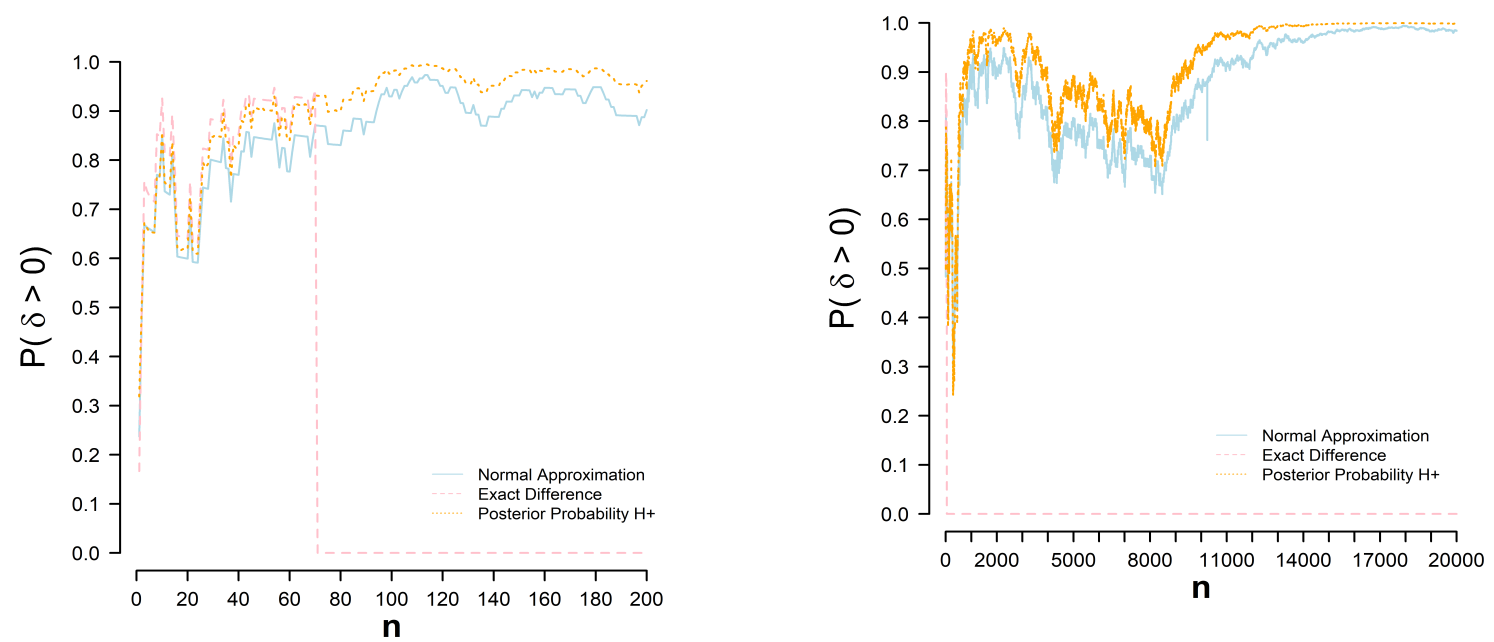

approximated by two normal distributions for sufficiently large $\alpha$ and $\beta$ values. Therefore, $\alpha$ and $\beta$ are used to calculate the parameters $\mu$ and $\sigma$ of the normal distributions, which then determine the distribution of $\delta$ (Appendix A).

However, both Figure B1a and Figure B1b show that the approximation is rather crude. A better way to calculate $\delta$ is to inspect the posterior probability of the hypothesis that the difference is positive rather than negative, that is, compare $\mathcal{H}_{+}$versus $\mathcal{H}_{-}$. To examine the entire probability distribution of the difference we can use:

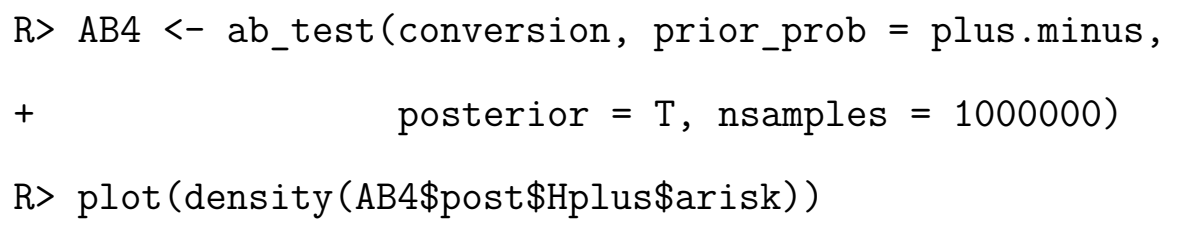

The code plots the posterior samples of the absolute risk under $\mathcal{H}_{+}$(see Figure B2). 
Comparing Figure $\mathrm{B} 2$ and Figure $4 \mathrm{a}$ as well as Figure $4 \mathrm{~b}$ confirms that this is a better method to calculate the exact difference as compared to the normal approximation.

As mentioned in the main text, it is also possible to monitor the difference over time with this technique. Figure 5 plots the sequential analysis of the posterior mean of the absolute risk, that is, the difference in success probabilities $\theta_{B}-\theta_{A}$. The data points for the plot can be produced with the following code:

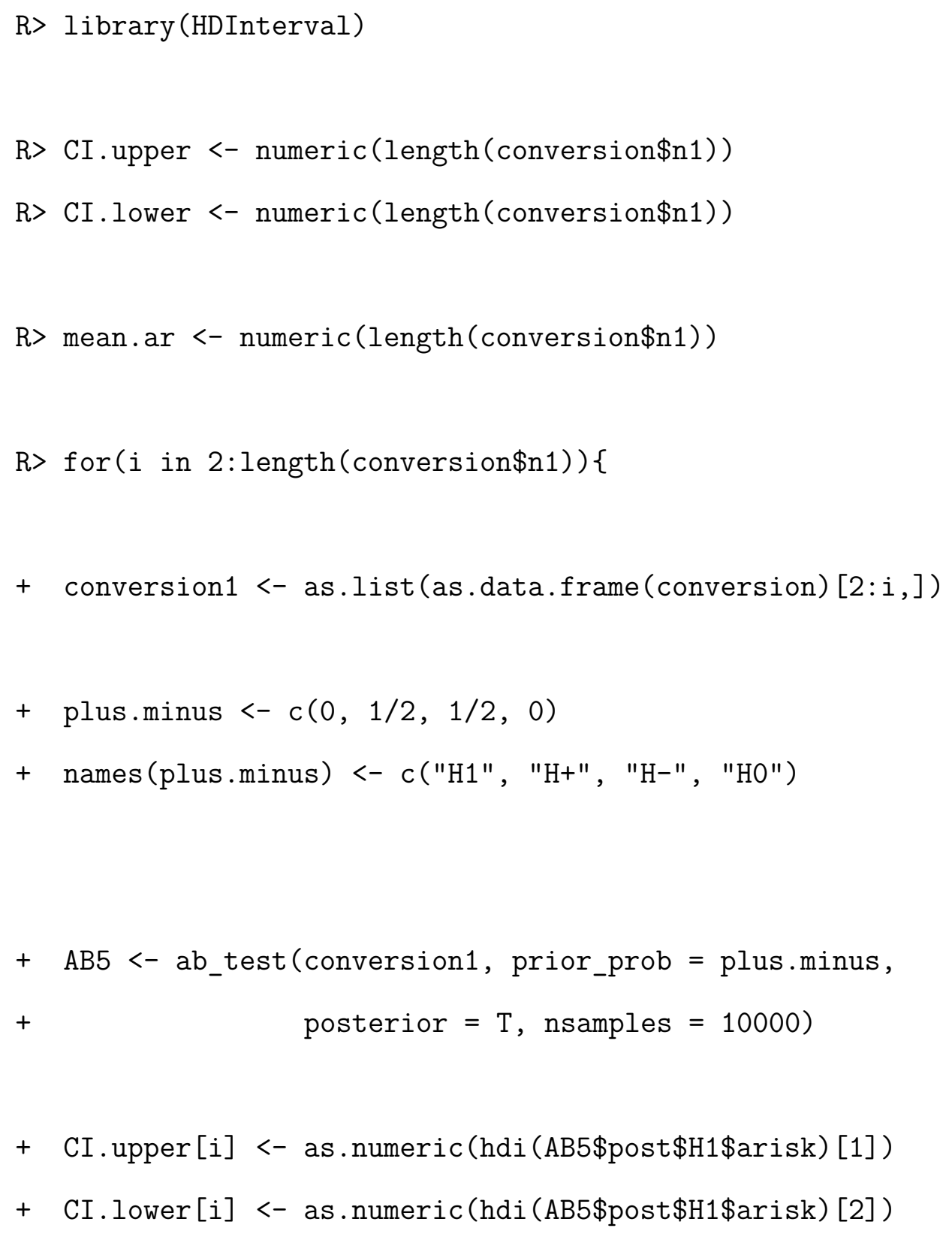


+ mean.ar $[i]<-$ mean (AB5\$post\$H1\$arisk)

\}

\section{Figure B2}

Posterior distribution of the absolute risk under $\mathcal{H}_{+}$. The absolute risk can be used to inspect the difference between the success probabilities of the two website versions.

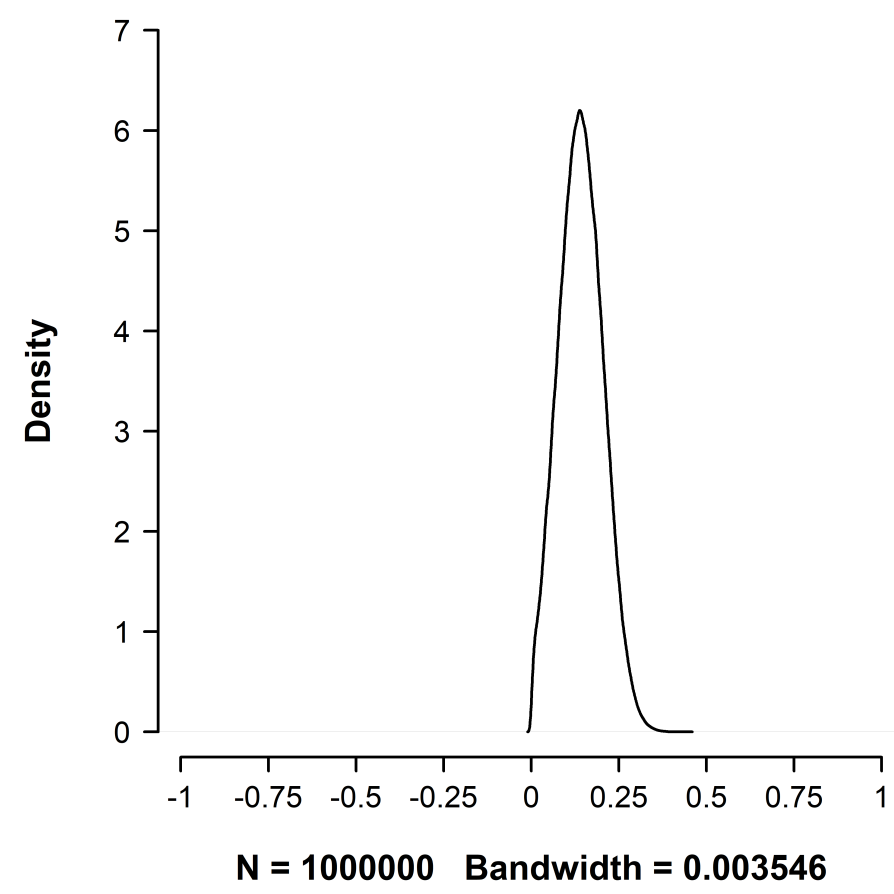

